\title{
EPS Sponsorship
}

It seems that there are still conference organizers who become aware too late that their meeting would have been eligible for EPS sponsorship had they applied in time. Organizers and potential participants both benefit from sponsorship, as EPS sponsored meetings are now recognized for their high standard, justifying the expenses incurred by the participants, and appearing in the coordinated calendar, designed to eliminate clashes of interest. Certain qualifications must, of course, be met. For a conference to be sponsored, there must be new material in the field meriting discussion, and the quality of speakers and papers should be high, as judged by a sound refereeing system. It must be located in Europe, with an

\section{University of Manchester Department of Physics}

Applications are invited for the post of RESEARCH FELLOW in the De-

partment of Physics. Candidates should have a higher degree and research experience in experimental nuclear physics. The successful applicant will assist in the research programme of the nuclear physics group and will also be expected to promote his own nuclear physics research interests. The group performs experiments at Manchester and at other accelerators in the U.K. and is also preparing for research on the new accelerator at the Daresbury Laboratory. The appointment is for two years in the first instance. Salary within the range $£ 3,660-£ 7,308$ p.a. Superannuation.

Letters of application should be addressed to :

Professor W.R. Phillips,

Department of Physics,

The University,

Manchester, M13 9PL,

and should also contain a curriculum vitae, together with the names of two persons to whom reference may be made. international committee to advise on the scientific programme. Participation should also be truly international, as regards both those attending the conference and the invited speakers, of whom more than half should come from outside the country in which the conference is staged. Moreover, the international advisory committee is required to see that there is a reasonable geographical distribution of the participants.

It is, in addition, essential that there be no restriction on race, colour, nationality etc. imposed by either the conference organizers or the authorities in the host country.

Organizers of conferences which seem to fall within the terms outlined above should write to the EPS Secretariat asking for a form of application for sponsorship. This form should be returned not less than one year before the conference is due to take place. This is in the interests of the organizers themselves, as only by working well in advance can the EPS Conference Committee ensure a logical programme of meetings that avoids overlap and gives maximum notice to potential participants, some of whom require at least a year's notice if they are to receive financial backing from the authorities in their own country.

If doubt exists whether a conference is eligible or not, contact the EPS Secretariat as soon as the essential data have been established.

\section{Guide to Entries}

Europhysics News publishes annually three Meetings Issues: in March, June and November. In the March issue the information received is given in full, whereas in the two subsequent issues some entries may be abbreviated and marked by an asterisk (*) indicating that full information can be found in the preceding Meetings Issue of Europhysics News.

The present issue contains details of future events about which notification was received at the EPS Secretariat in Geneva by May 1978. They are listed in date order in two categories - Conferences and Schools. Europhysics Conferences, i.e. meetings organized by EPS, its Divisions and Sections are printed in bold type and boxed; events sponsored by EPS are printed in bold type; Europhysics Study Conferences are marked by an $\boldsymbol{\theta}$ preceding the date.

Conference and school organizers who wish to have their meetings included in the next Meetings Issue of Europhysics News should apply to : European Physical Society,

P.O. Box 69 ,

$\mathrm{CH}-1213$ Petit-Lancy 2
Closing date for the Nov. 1978 issue is 17 Oct. Even if full information is not by then available, organizers are requested to send what details they already have.

\section{Key to Abbreviations}

\section{A : Application deadline}

$A b$ : Abstracts deadline

inv: attendance restricted to invited applicants

NP : no proceedings will be published

$P$ : Papers deadline (information on proceedings has not been received)

PP: proceedings will be published $\checkmark$ after the date indicates that there has been an important amendment to a previously published entry.

The information is given in the following order :

Date

Venue

Title

Contact for information

A : ... / Ab : ... / PP, NP or P : Papers deadline / Number of participants / inv. / languages other than English / Sponsorship / Conference fee. 


\section{Conferences}

\section{8}

19-28 July

Caracas, Venezuela

3rd Latin American Symposium: Magnetic Resonance and Hyperfine Interactions

3rd Symp. on MRHI, Apt. 59102, Los Chaguaramos Caracas 104

23-27 July Chicago, USA * 5th Annual Int. Conf. Microscopy (Inter-Micro 78) 24-26 July Newport Beach, CA, USA * Summer Computer Simulation Conf.

24-28 July

Rouen, France

* Int. Conf.: Conduction and Breakdown in Dielectric Liquids

28 July-8 Aug.

Helsinki, Finland URSI General Assembly

31 July-4 Aug. Windsor, Ont., Canada * 4th Int. Conf. on Spectral Line Shapes

1-5 Aug. Uppsala, Sweden

* IAU Symposium: Refractional Influences in Astrometry and Geodesy

1-9 Aug. Toronto, Canada * 9th Int. Congress on Electron Microscopy

3-12 Aug. Warsaw, Poland * 11 th Int. Congress of Crystallography

7-11 Aug.

Toronto, Canada

6th Int. Heat Transfer Conference

K. Charbonneau, 6th Int. Heat Transfer Conf c/o National Research Council of Canada Ottawa, Ont., Canada K1A OR6

Ab: 1.4 .77 / P: 15.7 .77

7-12 Aug.

Uppsala, Sweden 4th European Regional Meeting in Astronomy: Stars and Star Systems

B. Westerlund, Astronomical Observatory, Box 515, S-75120 Uppsala

A: 1.6.78/Ab: 15.5.78/400/IAU / sKr. 115. -

9-12 Aug.

Groningen, NL

* Formation of Images from Spatial Coherence

Functions in Astronomy

10-12 Aug.

Troy, NY, USA

* Int. Symposium on Photopion Nuclear Physics

13-16 Aug.

Kozubnik, Poland

* Conference on Applied Crystallography

14-15 Aug.

Cracow, Poland

Diffraction Profile Analysis

Open Meeting: Commission on Neutron Diffraction A. Oleś, Institute of Physics and Nuclear Techniques, A.G.H., Al. Mickiewicza 30, PL-30-059 Cracow PP

14-17 Aug.

Wroclaw, Poland

* Symposium on Crystal Structure and Bonding of Metal Complexes and Biologically Important Metal Compounds

14-18 Aug.

Singapore

Int. Meeting on Frontier of Physics

K.K. Phua, Dept. of Physics, Nanyang University, Jurong Road, Singapore 22

14-18 Aug. Blindern, Norway

* Physics of Solar Prominences

Pécz, Hungary

14-19 Aug. ce (and School)

15-23 Aug.

* International Congress of Mathematicians

17-22 Aug.

Riga, USSR

6th Int. Conf. on Atomic Physics

Canberra, Australia

18 Aug.-2 Sept.

* Int. Conference on Nuclear Interactions

20-25 Aug.

* 3rd Euroanalysis Conference

Dublin, Ireland

* For full details please see March 1978 issue of Europhysics News
San Diego, CA, USA

20-25 Aug.

ion Engineering Conf.

20-26 Aug.

Rochester, NY, USA

* Int. Congress of Photographic Science 1978

(9) 21-23 Aug.

Vejle, Denmark

2nd European Study Conf. on Low Energy Molecular Collisions (MOLEC 2)

U. Buck, Abt. Atom- und Molekül-Physik, Max-Planck-Inst. f. Strömungsforschung, Postfach 867, D-3400 Göttingen

A: 1.5.78 / Ab: 1.5.78 / NP / 100/inv. / dKr. 1000.

21-24 Aug.

Zurich, Switzerland

* 2nd World Hydrogen Energy Conference

21-26 Aug.

Tallinn, USSR

20th Congress Ampère: Magnetic Resonance and Related Phenomene

T.A. Saluvere, Academy of Sciences of the Estonian SSR, Lenini puiestee 10, Tallinn 200001

A: 1.6.78 / Ab: 15.4.78 / P: 1.6.78/800/ US $\$ 40$. -

22-25 Aug.

Melbourne, Australia

* Australian Conference on Electron Spectroscopy

22-25 Aug.

Prague, Czechoslovakia

8th Int. Symposium: IMEKO Technical Committee on Photon-Detectors

22-28 Aug. Tbilisi, USSR

* 3rd IUTAM Symposium on Shell Theory

23-29 Aug. Grenoble, France

* LT 15 - 15 th Int. Conf. on Low Temperature Physics

23-30 Aug. Innsbruck, Austria

7th Int. Conf. on Plasma Physics and Controlled Nuclear Fusion Research

23-30 Aug.

Tokyo, Japan

* 19th Int. Conf. on High Energy Physics

24-30 Aug.

Graz, Austria

- 8th Int Conf.: Few Body Systems and Nuclea

Forces

25-29 Aug.

IMU/IUTAM Symposium: Group Theoretical Methods in Mechanics

28-30 Aug. Manchester, UK

Ion Implantation and Ion Beam Techniques in Corrosion

Dr. Proctor, UMIST, POB 88, Manchester M60 10D

28-31 Aug.

San Diego, CA, USA

22 nd Int. Symposium and Instrument Display

Society of Photo-Optical Instrumentation Engineers, POB 10, Bellingham, WA 98225

Ab: 7.7 .78 / P: 7.7 .78

28 Aug.-1 Sept.

* Meteorological Optics (28.8-29.8)

Atmospheric Spectroscopy (30.8-1.9)

28 Aug.-1 Sept. Louvain-la-Neuve, Belgium * IUTAM Symp.: Non-Newtonian Fluid Mechanics

28 Aug.-1 Sept.

Sendai, Japan

* Int. Conf. on X-Ray and XUV Spectroscopy

28 Aug.-1 Sept.

Kyoto, Japan

" 8th Int. Conf.: Applications of Mössbauer Effect

28 Aug.-2 Sept. Canberra, Australia Int. Conference on Nuclear Interactions J.O. Newton, Dept. of Nuclear Physics,

Australian National Univ., РОВ 4, Canberra ACT 2600 IUPAP

28 Aug.-3 Sept. Tutzing nr. Munich, FRG * IUTAM Symposium: Metal Forming Plasticity

29-31 Aug.

Jülich, FRG Int. Conf. on Neutron Scattering and Magnetism
Tagungsbüro KFA Jülich, Pf. 1913, D-5170 Jülich

30 Aug.-1 Sept.

College Park, MD, USA

Stellar Interferometry

30 Aug.-1 Sept. $\begin{array}{r}\text { Southampton, UK } \\ \text { * Int. Conf.: Recombination in Semiconductors }\end{array}$

Southampton, UK IUPAP
30 Aug.-2 Sept.

Grenoble, France

3rd Int. Conf. on Electronic Structure of Actinides

J.M. Fournier, DRF-CENG, $85 \times$ F-38041 Grenoble Cedex

A: 15.6.78 / Ab: 1.4.78 / PP: 15.7.78 / 100 / English, French, German / FF 600. - incl. accommodation

G 31 Aug.-9 Sept.

Les Houches, France

EPS Study Conference on Solid ${ }^{3} \mathrm{He}$ and ${ }^{4} \mathrm{He}$ and Liquid ${ }^{3} \mathrm{He}$

M.T. Béal-Monod, I.L.L., Ave. des Martyrs,

B.P. 156, Centre de Tri,

F-38042 Grenoble Cedex

A: 31.8 .78 / NP / 70 / inv. / FF 420.

1-5 Sept.

Trieste, Italy

Workshop on Drift Waves in Hot Plasmas

The Deputy Director, International Centre for

Theoretical Physics, POB 586, I-34100 Trieste inv.

3-9 Sept.

Kyoto, Japan

6th International Biophysics Congress

4-5 Sept.

Meeting on Exotic Resonance

Hiroshima, Japan

M. Yonezawa, Department of Physics, Hiroshima University, Higashisendamachi, Hiroshima 730 A: 20.6.78 / Ab: 1.7 .78 / PP

4-6 Sept.

Oxford, UK

Applications of Machine-Aided Image Analysis D.J. Stanley, B 521, AERE Harwell, OX11 ORA A: 15.7 .78 / Ab: 17.4.78 / PP: 1.8.78 / 120

4-6 Sept

Grenoble, France

COMPUMAG: Computation of Magnetic Fields

COMPUMAG Secretariat, Lab. d'Electro-

technique, ENSEGP, Domaine Universitaire B.P. 46, F-38406 St. Martin d'Heres

Ab: 1.3 .78 / 250 / FF 400. -

4-7 Sept.

St. Pierre de Chartreuse, F

Physique des Terres Rares à I'Etat Métallique

R. Lemaire, Laboratoire de Magnétisme CNRS, 166X, F-38042 Grenoble Cedex

A: July 1978 / P: 30.6.78 / 100 / inv. / FF 250.-

4-7 Sept.

Rorschach, Switzerland

1 st Int. Topical Meeting on Muon Spin Rotation ( $\mu$ SR)

Conference Secretariat, SIN, CH-5234 Villigen

A: 15.6.78 / Ab: 15.6.78/ PP / 70 / Sfr. 125.-

4-8 Sept.

Budapest, Hungary

Ion Beam Modification of Materials

4-8 Sept. Dubrovnik, Yugoslavia

Quasi One-Dimensional Conductors

S. Barisic, Institute of Physics, University, Bijenicka cesta 46, POB 304, YU-41001 Zagreb

A: 31.5.78 / Ab: 31.5.78 / PP: 30.10 .78 / $150 /$ US $\$ 70$. -

4-8 Sept

Villeurbanne, F

Fast lon Spectroscopy - 5th Int. Conf. on BeamFoil Spectroscopy

Jean Desesquelles, Laboratoire de Spectrométrie lonique et Moléculaire, Université de Lyon I, Campus de Doua,

F-69621 Villeurbanne

PP: June 78 /150 / English, French / FF 350.-

4-8 Sept.

Trieste, Italy

1st Seminar on Solar Energy Storage: Thermal Storage

The Deputy Director, International Centre for Theoretical Physics, POB 586, I-34100 Trieste A: 31.7 .78

4-8 Sept. York, UK

* 4th Conf. on Physical Organic Chemistry

4-8 Sept.

London, UK

* 7th Symposium on Photoelectronic Image Devices

4-8 Sept.

Padua, Italy

* 10th Symposium on Fusion Technology (SOFT)

4-8 Sept.

Edinburgh, UK

* 14th Int. Conf. on the Physics of Semiconductors

4-8 Sept. Prague, Czechoslovakia

* 19th Int. Conf. on Coordination Chemistry

4-9 Sept. Bangalore, India

* 6th Int. Conf. on Raman Spectroscopy

IUPAP 
5-8 Sept.

Avignon, France

2nd Int. Coll.: Electron Beam Melting and Welding

6-8 Sept.

Int. Conf.: Analysis and Optimization of Oxford, UK

Institute of Mathematics and its Applications, Maitland House, Warrior Square, A: 6.8 .78

6-8 Sept.

Reading, UK

Annual Conf. of British Assoc. for Crystal Growt R.A.M. Scott, Materials Science and Physics, Thames Polytechnic, Wellington Str., London SE18 6PF A: $21.7 .78 / A b: 7.7 .78$

6-8 Sept.

Bad Honnef, FRG

Seminar: Optische Eigenschaften magnetisch geordneter Materialien

J. Schneider, Inst. für Angew. Festkörperphysik Ekkertstr. 4, D-7800 Freiburg i. Br.

7-9 Sept. Rome, Italy * Perspectives on Fundamental Physics

10-17 Sept.

Madrid, Spain

* Congress of International Commission for Optics

11-13 Sept.

Evanston, IL, USA

IUTAM Symposium on Variational Methods in Mechanics

S. Nemat-Nasser, The Technological Institute

Northwestern University, Evanston, IL 60201

inv. / IUTAM

11-14 Sept.

Liège, Belgium

Dense Random Packing - Amorphous Metals and Small Metallic Clusters

11-14 Sept.

Nice, France

10th Int. Conf. on Defects and Radiation Effects in Semiconductors

11-14 Sept.

Liverpool, UK

* 5th Int. Conf.: Gas Discharges

Oxford, UK

11-15 Sept.

Applications of High Magnetic Fields in Semiconductor Physics

11-15 Sept.

Groningen, NL

FLOMEKO 1978 - IMEKO - Conf. On Flow Measurement of Fluids

FLOMEKO 1978, РОВ 19, Groningen

A: Aug. 77 / Ab: 1.9.77 / PP: 31.12.77 / 300 hFl. 400, - hFl, 100, - students

11-15 Sept. Bad Honnef, FRG * Workshop on High-Current-Electrode and HollowCathode Phenomena

11-15 Sept. Loughborough, UK

4th Int. Thin Films Congress - Thin Film Properties in Relation to Their Structure

C.J. Todd, Post Office Research Centre, Martlesham Heath, Ipswich, IP5 7RE

A: 7.8.78/ Ab: 1.4.78/PP: 1.8.78/500 / IUPAP, IUVSTA

11-15 Sept.

Kiel, FRG

6th European Cosmic Ray Symposium

W. Enge, Inst. f. Reine u. Angewandte Kernphysik, Christian-AlbrechtsUniversität, Olshausenstr. 40/60 Universität, Olshausenstr. 40/6C
Gebäude N 20 a, D-2300 Kiel

A: 1.7.78/Ab: 1.7.78/NP / 120 / DM 40.-

11-15 Sept.

Montpellier, France

8th European Solid State Device Research Conf. - ESSDERC 78

3rd Symposium on Solid State Device Technology - SSDT 78

J.P. Nougier, Université des Sciences et Techniques du Languedoc, Centre d'Etudes d'Electronique des Solides,

d'Electronique des Solides,
F-34060 Montpellier Cedex

Ab: 5.5.78 / PP: 15.8.78 / 300 / FF 550.FF 650.- after 15.7.78

11-16 Sept.

Austin, TX, USA

7th Int. Group Theory Colloquium - Integrative Conf. on Group Theory and Mathematical Physics

11-23 Sept.

Trieste, Italy

Séminaire sur l'énergie solaire

The Deputy Director, International Centre for

Theoretical Physics, POB 586, I-34100 Trieste A: 30.6.78/French

*or full details please see March 1978 issue of Europhysics News
Liverpool, UK

-13 Sept.

nt Diffusion in the Environment

Institute of Mathematics and its Applications, Maitland House, Warrior Square,

Southend-on-Sea, Essex, SS1 2JY

A: 14.8 .78

12-14 Sept.

Olloque: Equipements de Photogrammétrie Analytique et de Télédétection

12-14 Sept.

Vienna, Austria

* IAU Colloquium: Modern Astrometry

12-14 Sept.

Brighton, UK

Science and Security

La Jolla, CA, USA

$A b: 7.7 .78$

12-15 Sept. Nottingham, UK

3rd EPS Computational Physics Conf. Computational Atomic and Molecular Physics

R.L. Hudson, Mathematics Dept., Univ. of Nottingham, University Park, Nottingham NG7 2RD

A: 29.8.78 / Ab: 15.7.78 / PP / 150

£ 40. - approx.

12-15 Sept.

Genoa, Italy

4th Europ

(ECOC)

13-15 Sept.

* Int. Conf.: Measurement of Force and Mass

13-15 Sept.

Structure and Motion in Molecular Liquids

Mrs. Y.A. Fish, The Chemical Society,

Burlington House, London W1V OBN

A: 16.8.78 / Ab: 31.10 .77 / PP: 1.5 .78 / 150

14-16 Sept.

Bratislava, Czechoslovaki Industrial Applications of Soft Magnetic Alloys J.E. Thompson, Wolfson Centre for Magnet

Technology, Univ. College, 30 The Parade Rd. Cardiff CF2 3AD, UK

17-24 Sept.

Kupari, Yugoslavia

* Seminar: New Approaches to the Teaching and Learning of Physics

18-19 Sept.

Dym Pressure Sources

M. Outred, Dept. of Physics, Polytechnic of North London, Holloway Road, London N7 8DB

\section{8-20 Sept. Essen, FRG \\ SCAMPIG - 4th Europhysics Sectional Conference on Atomic and Molecular Physics in lonized Gases \\ H.F. Döbele, Fachbereich Physik, Universität Essen, Postfach 6843. D-4300 Essen \\ A: 15.8.78/Ab: 1.7.78/100/DM 100.-}

18-21 Sept.

Aberdeen, UK

Upper Atmosphere Studies by Optical Methods M. Gadsden, Dept. of Natural Philosophy, University of Aberdeen, Aberdeen AB9 2UE

18-21 Sept.

Amsterdam, NL (ESSCIRC 78 ,

18-21 Sept.

Blooming their

$$
\begin{aligned}
& \text { th Int. Conf. on } \\
& \text { Applications }
\end{aligned}
$$

18-21 Sept.

Coventry, UK

Kinetics of Bimolecular Ion Molecule Reaction K.R. Jennings, Dept. of Chemistry and Molecular Sciences, University of Warwick. NP

$$
\text { Coventry CV4 7AL }
$$

18-21 Sept.

Leipzig, GDR

Polymerphysik

Physikalische Gesellschaft der DDR, Am Kupfergraben 7, DDR-108 Berlin
18-22 Sept.

Upton, L.I.

* 3rd Int. Symposium on Neutron Capture Gamma Ray Spectroscopy and Related Topics

18-22 Sept.

Liblice, Czechoslovakia

5 th Int. Seminar on High Resolution Infrared Spectroscopy

J. Heyrovsky, Inst. of Phys. Chem.

Czechoslovak Academy of Sciences,

Flemingova nam. 2, CS-16000 Praha 6

18-22 Sept.

Gaithersburg, MD, USA

10th Materials Research Symposium:

Characterization of High Temperature Vapors and Gases

19-21 Sept.

Hamburg, FRG

PETRA Topical Meeting: Heavy Quarks and Leptons Fritzsch, Dept. of Physics, University of Wuppertal D-5600 Wuppertal

19-22 Sept.

8th Europhysics Conf. on Macromolecular Physics: Structural Aspects common to Synthetic and Biological Macromolecules

A. Keller, H.H. Wills Physics Laboratory, Bristol BS8 1TL

A: 14.7.78 / Ab: 1.4.78 / NP / 120

19-23 Sept.

Siena, Italy

64th Congress of Italian Physical Society

Segretaria della Società Italiana di Fisica, Via L. degli Andalò 2, I-40124 Bologna

A: 30.6.78 / Áb: 30.6.78 / L. 6000.- mbrs.;

L. 10000 - non-mbrs.

20-22 Sept.

London, UK

Atomic Collision Processes

A.C.H. Smith, Dept. of Physics and Astronomy

University College, London WC1E 6BT

20-22 Sept.

Antibes, France

4th Coll. on Solid Surface Physics and Chemistry: Gas-Solid and Liquid-Solid Interface

Mme J. Fauvet, Société Française du Vide 19 rue du Renard, F-75004 Paris

A: 28.4.78 / PP: 29.6.78 / English, French /

FF 792. - incl. proc.

20-22 Sept.

Edinburgh, UK

Physics and Chemistry of Laser Induced Processes in Molecules

R.G. Harrison, Heriot-Watt University, Riccarton, Currie,

Edinburgh EH14 4AS

A: 15.7.78/Ab: 31.5.78/ PP / $200 /$

$f$ 45. - approx. incl. proc.

20-22 Sept.

Symposium: Penetration of Atomic Particles through Matter

20-22 Sept.

St, Andrews, UK

* 2nd Int. Meeting on Solid Electrolytes

20-23 Sept.

Bath, UK

25-27 Sept.

Salzburg, Austria

12. Spectrometertagung

W. Schwarz, Vereinigte Edelstahlwerke, Kapfenberg, Versuchs-

A-8605 Kapfenberg

* Applied Superconductivity Conference

25-28 Sept.

Brighton, UK

* 11 th Int. Symposium on Power Sources

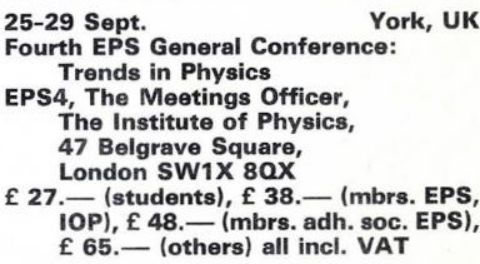

EPS4, The Meetings Officer, The Institute of Physics,

47 Belgrave Square, London SW1X $80 \mathrm{X}$

£ 27. - (students), £ 38.- (mbrs. EPS IOP), $£$ 48. - (mbrs. adh. soc. EPS), $£ 65$. - (others) all incl. VAT

25-29 Sept.

Fontevrault, France

Int. Meeting: Nonlinear Behaviour of Molecules, Atoms and lons in Electric, Magnetic or

Electromagnetic Fields 
C. Troyanowsky, Secrétaire général, Société de Chimie physique, 10 rue Vauquelin, F-75005 Paris

25-29 Sept. Innsbruck, Austria * General Conf. on Physics (Austrian Phys. Soc.)

25-29 Sept. Harwell, UK

* Int. Conf.: Neutron Physics and Nuclear Data for Reactors and other Applied Purposes

25-29 Sept. London, UK

* 7 th Int. Broad BC 78)

26-28 Sept.

Vienna, Austria

Aerosols in Science, Medicine and Technology Dynamics and Measurements of Ultrafine Aerosols

R. Jaenicke, Gesellschaft für Aerosolforschung Max-Planck-Institut, POB 3060 .

D-6500 Mainz, FRG

27-29 Sept.

Int. Pulvermetallurgische Tagung

Zentralinst. für Festkörperphysik und Werkstoffforschung, Helmholtzstr. 20. DDR-8027 Dresden

29-30 Sept.

* 3rd Int. Conf. on Bio-Impedance

Bologna, Italy

1-4 Oct.

Biophysik-Tagung 1978

UIm, FRG

E. Sackmann, Abt. f. Experimentelle Physik III, Universität Ulm, Postfach 4066, D-7900 Ulm

1-8 Oct.

Dubrovnik, Yugoslavia

Astronautics for Peace and Human Progress

International Astronautical Federation 250 rue Saint-Jacques, F-75005 Paris Ab: $5.4 .78 / P P$

2-4 Oct.

West Berlin, FRG

8. Jahrestagung der Gesellschaft für Informatik (G)

2-5 Oct.

Le Chesnay, France

2 nd Int. Symposium on Operating Systems

RIA, Service des Relations Extérieures, Domaine de Voluceau, B.P. 105, Rocquencourt, F-78150 Le Chesnay

Ab: 24.2.78/P: 1.7.78/English, French

Stresa, Italy
SEAS Anniversary Meeting (Computer Applications,

2-6 Oct.
SEAS Anniversary Meeting (Computer Applications, Networking)

SHARE European Association, Toernooiveld, Nijmegen, The Netherlands

A: 1.9.78/Ab: 1.7.78/P: 15.9.78/300/hFl. 200.-

2-7 Oct.

Trieste, Italy

Recent Progress in Many-Body Theories

Deputy Director, International Centre for Theoretica inv.

3-4 Oct. Physics, POB 586, I-34100 Trieste

London, UK

Colour Measurement and its Application

e Meeting Officer, The Institute of Physics,

47 Belgrave Square, London SW $1 \times 80 X$

3-6 Oct.

West Berlin, FRG

42. Physikertagung der DPG

West Berlin, FRG Haberland, Institut f. Festkörperphysik I, Strasse
des 17. Juni, 135, D-1000 Berlin 12

3-7 Oct. Modra-Harmonia, Czechoslovakia Hadron Structure '78

Jan Pisut, Dept. of Theor. Phys., Comenius University, Mlynska Dolina, CS-81631 Bratislava

5-8 Oct.

Brigue, Switzerland

158e Assemblée annuelle: Société helvétique des sciences naturelles

S. Escher, Belalpstrasse 2, $\mathrm{CH}-3900$ Brig-Glis A: 10.9 .78 / PP: 31.10 .78 / English, French, German

6-7 Oct. Brigue, Switzerland Autumn 1978 Meeting of Swiss Physical Society Société Suisse de Physique, Secrétariat B.S.P. Université, $\mathrm{CH}-1015$ Lausanne

Ab: 15.8.78 / PP / 300 / English, French, German, Italian

9-10 Oct.

London, UK

Atmospheric Sensing with Lasers

The Meetings Officer. The Institute of Physics, 47 Belgrave Square, London SW 1 X $80 X$ Ab: 10.7 .78

9-11 Oct.

San Francisco, CA, USA

6th Semiconductor Laser Conference

T.L. Paoli, Bell Laboratories, 600 Mountain Ave Murray Hill, NJ 07974
9-12 Oct.

Kyoto, Japan

Int. Workshop on Electric Charges in Dielectrics Y. Wada, Dept. of Applied Physics, University of Tokyo, Bunkyo-ku, Tokyo 113

10-12 Oct.

Venice, Italy

European Co-operation in Informatics (ECl 78)

VDE-Zentralstelle Tagungen, Stresemannallee 21. D-6000 Frankfurt/Main 70, FRG

10-13 Oct.

Utrecht, NL

4th European Electro-Optics Conf. \& Exhibition

Secretariat EEO 78, Miss D.H. Friloux, Arthur D. Little S.A., 90 ave. des ChampsElysées, F-75008 Paris

A: 1.9.78/PP: 1.3.78/300/ hFI. 400.-

16-20 Oct.

Nice, France

Evolution of Planetary Atmospheres and Climatology of the Earth

Centre National d'Etudes Spatiales, Dépt. des Affaires Universitaires, 18 avenue Edouard-Belin, F-31055 Toulouse Cedex

Ab: 15.4.78 / 250 / English, French / FF 400.-

\section{7-19 Oct.}

Kiamesha Lake, NY, USA

10th National SAMPE Technical Conf.: Materials Synergisms

SAMPE, POB 613, Azusa, CA 91702

$A b: 1.2 .78 / P: 1.6 .78$

17-20 Oct.

Buffalo, NY, USA

31st Annual Gaseous Electronics Conference

David M. Benenson, Dept. of Electrical Engineering State University of New York at Buffalo

4232 Ridge Lea Road, Amherst, NY 14226

Ab: 11.8 .78

17-21 Oct.

Tashkent, USSR

nt. Symposium on Macromolecular Chemistry

K. Andrianov, Polymer Scientific Council, Academy

of Sciences of USSR, Vavilov Street 32.

IUPAC

18-20 Oct.

Symposia: Nuclear Science: Scintillation and Semiconductors; Nuclear Power Systems

Louis Costrell, US Department of Commerce National Bureau of Standards,

Washington, DC 20234

23-25 Oct.

Frontiers of Education

Lake Buena Vista, FL, USA

E.R. Chenette, Dept. of Electrical Engineering. Univ. 500 of Florida, Gainesville, FL 32611

23-25 Oct.

Brighton, UK

Mini \& Micro Forum

Online, Cleveland Road, Uxbridge, UB8 2DD

Ab: $20.2 .78 / P: 31.7 .78$

25-28 Oct.

Argonne, IL, USA

3rd Int. Symposium on High Energy Physics with Polarized Beams and Polarized Targets

G.H. Thomas, HEP 362, Argonne National Laboratory, Argonne, IL 60439

30 Oct.-3 Nov.

San Francisco, USA

Annual Meeting: Optical Society of America

J.W. Quinn, Optical Society of America, 2000 Street, N.W. Suite 620. Washington, DC 20036 Ab: 7.7 .78 / US \$ 45. -

30 Oct.-4 Nov. Colorado Springs, CO, USA Meeting of Plasma Physics Division (APS)

American Physical Society, 335 E. 45th Street, New York, NY 10017

Ab: 15.8 .78

1-3 Nov.

Asilomar, CA, USA

Meeting of the Nuclear Physics Division (APS)

The American Physical Society, 335 E. 45th Street New York, NY 10017

6-8 Nov.

Denton, TX, USA

5 th Conference on the Use of Small Accelerators Jerome L. Duggan, Physics Dept., North Texas State University, N.T. Box 5368, Denton, TX 76203

G. 7-10 Nov.

Workshop on Future Solar Optica

o. Engvervations - Needs and Constraints

physict

Blindern, Oslo 3, Norway

A: $31.5 .78 / 30 /$ inv.

14-17 Nov.

Cleveland, Ohio, USA

Magnetism and Magnetic Materials

J.J. Rhyne, Reactor Radiation Div., National Bureau PP

15-18 Nov.

Washington, DC, USA

3rd Int. Conf. on Electrophotography (SPSE)

R.H. Wood, Society of Photographic Scientists and Engineers, $1411 \mathrm{~K}$ Street, NW, Washington, DC 20005

16-17 Nov.

London, UK

Deeting on Solar Physics

The Royal Society, 6 Carlton House Terrace, London SW1Y 5AG

20-22 Nov.

Los Angeles, USA

Meeting of Fluid Dynamics Division (APS)

American Physical Society, 335 E. 45th Street, New York, NY 10017

27 Nov.-1 Dec.

Hamilton, New Zealand

IAU Colloquium: Changing Trends in Variable Star Research

F.M. Bateson, 18 Pooles Rd, Greerton, Tauranga, NZ $100 /$ inv. / IAU

28-30 Nov.

London, UK

Int. Conf.: Antennas and Propagation

IEE Conf. Dept., Savoy Place, London WC2R OBL Ab: 31.3.78/PP: 21.7.78/URSI

28 Nov.-1 Dec.

San Francisco, USA

Annual Meeting of the American Vacuum Society

335 E. 45th Street, New York, NY 10017

4-6 Dec.

Washington, DC, USA

Int. Electron Devices Meeting

Technical Activities Board, IEEE, 345 E. 47th Street, New York, NY 10017

4-8 Dec.

Gaussig nr. Dresden, GDR

2nd Int. Seminar on Magnetism

K. Elk, Hochschule f. Verkehrswesen 'Friedrich List', Wissenschaftsbereich Physik, Postfach 103, DDR-8072 Dresden

50 / English, Russian / M 150.-

5-8 Dec.

Wellington, New Zealand Asian-South Pacific Regional Meeting in Astronomy B.M. Lewis, P.O. Box 2909, Wellington, NZ

NP / 150 / English, French / IAU

18-19 Dec.

London, UK Pulsed Nuclear Magnetic Resonance in Solids Mrs. Y.A. Fish, Faraday Division, The Chemical Society, Burlington House, London W1V OBN A: 20.11 .78 / Ab: 10.10 .77 / PP: $1.8 .78 / 150$

18-19 Dec.

London, UK

Materials in a Marine Environment

The Meetings Officer, The Institute of Physics,

47 Belgrave Square, London SW1X $80 X$

Ab: 31.5 .78

27-29 Dec.

Los Angeles, USA

General Meeting of American Physical Society American Physical Society, 335 E. 45th Street, New York, NY 10017

27-29 Dec.

Moscow, USSR

Int. Conference on Collective Phenomena

Brailovsky, Vernadski Prospekt 99, korp. 1 apt. 128 , Moscow 119285

\section{9}

\section{3-5 Jan.}

Coventry, UK

16th Annual Solid State Physics Conference

47 Belgrave Square, London SW1X 80X

4-5 Jan.

Bangor, UK

Laser Plasma Interactions

The Meetings Officer, The Institute of Physics,

47 Belgrave Square, London SW $1 \times 80 X$

8-12 Jan.

Geilo, Norway

Nordic Meeting on Intermediate Energy Physics Sven Kullander, Gustaf Werner Institute, Box 531, 50 S-75121 Uppsala, Sweden 


\section{European Physical Society}

POB 69

CH-1213 Petit-Lancy 2

Tx. 23455 alarm ch

Cables: Europhys Genève

Executive Secretary

G. Thomas

\section{Individual Ordinary Members}

In June 1978 the total number of Individual Ordinary Members belonging to the European Physical Society totalled 3234. The names of the nine delegates to Council of these members are shown overleaf.

\section{Honorary Members}

$\begin{array}{ll}\text { G. Bernardini } & \text { G. Szigeti } \\ \text { Pisa } & \text { Budapest } \\ \text { Italy } & \text { Hungary }\end{array}$

\section{Fellows}

\section{H.B.G. Casimir}

Heeze

The Netherlands

\section{Member Organizations}

(X) $Y$

$X=$ No. of members of the Organ.

$\mathrm{Y}=$ No. of delegates to Council

\section{Ampere Group (254) 1 \\ Del. E.R. Andrew \\ Pres. E.R. Andrew \\ Department of Physics, University \\ of Nottingham, University Park \\ Nottingham NG7 2RD UK \\ Sec-Gen. G.J. Béné \\ Université de Genève, Section de \\ Physique, boulevard d'Yvoy 32 \\ $\mathrm{CH}-1211$ Geneva 4 \\ T. (22) 219355}

Austrian Physical Society (487) 1

Del. H. Zingl

Pres. P. Weinzier

University of Vienna

Strudlhofgasse 4

A-1090 Vienna

Sec. H. Kirchmayr

Institute for Experimental Physics

Karlsplatz 13

A-1040 Vienna

T. (222) 653785

Belgian Physical Society (360) 1

Del. J. Lemonne

Pres. J. Lemonne

Vrije Universiteit Brussel

Pleinlaan 2

B-1050 Brussels

Sec-Gen. P. Fettweis

CEN/SCK

Boeretang 200

B-2400 Mol

T. (14) 311801

Bulgarian Acad. of Sciences (105) 1

Del. A. Datzeff

Del. A. Datzeff

Sec-Gen. G. Bliznakov

Foreign Relations Department

7 November str. No. 1

Sofia BG

T. 877731

Danish Physical Society (350) 1

Del. A.R. Mackintosh

Pres. A.R. Mackintosh

H.C. Orsted Institute

Universitetsparken 5

DK-2100 Copenhagen 0
Danish Phys. Soc. Contd.

Sec. J. Kjems

Physics Department, AEC Est. Ris $\varnothing$ DK- 4000 Roskilde

T. (3) 355101

Eötvös Lorand Physical Society

(610) 2

Dels. I. Abonyi

G.J. Csika

Pres. G. Marx

Dept of Atomic Physics

Eötvös Lorand University

Puskin u 5-7

H-1088 Budapest

Sec-Gen. G.J. Csikai

Eötvös Lorand Fizika

Tarsulat, POB 240

$\mathrm{H}-1368$ Budapest

T. 227040 Tx. 225369

Finnish Physical Society (559) 2

Dels. K. Eskola

$$
\text { V. Ruuskanen }
$$

Pres. V. Ruuskanen

University of Jyväskylä

Department of Physics

Nisulankatu 78

SF-40720 Jyväskylä 72

T. (941) 291211

\section{Sec. R. Sälke}

Department of Physics

University of Helsinki

Siltavuorenpenger 20

SF-00170 Helsinki 17

T. (90) 650211

\section{Dept. of Gen. Physics \\ and Astronomy, \\ USSR Acad. of Sciences (2000) 4 \\ Dels. S. Kapitza \\ I. Krilov \\ K.K. Rebane \\ G.A. Smolensky \\ Sec-Gen. A.M. Prokhorov \\ Sci-Sec. S. Markyanov \\ USSR Academy of Sciences \\ Leninskyi Prospekt 14 \\ Moscow B-71 USSR \\ T. 2322910}

\section{Executive Committee}

President : A. Zichichi

CERN, EP Division

$\mathrm{CH}-1211$ Geneva 23

T. (22) 833196

Vice-President : S. Kapitza Institute for Physical Problems

Vorobyevskoe Shosse 2

USSR-117 334 Moscow

T. 1373247

Secretary: M. Guenin

Université de Genève, Dépt. de

Physique Théorique, 32 blvd d'Yvoy

$\mathrm{CH}-1211$ Geneva 4

T. (22) 219355

Vice-Secretary: Edith A. Mueller Observatoire de Genève

$\mathrm{CH}-1290$ Sauverny

T. (22) 552611

\section{Treasurer: L.A.A. Thomas}

The General Electric Co. Ltd.

Hirst Research Centre, East Lane

Wembley HA9 7PP, Middlesex UK

T. (904) 59861

Vice-Treasurer: A.R. Mackintosh

Physics Laboratory

H.C. Orsted Institute

Universitetsparken 5

DK-2100 Copenhagen 0

T. (1) $353133 / 215$

Members :

A. Lösche

Sektion Physik der Karl-Marx-Uni-

versität, Linnestrasse 5

DDR-701 Leipzig

T. (41) 68580

W. Martienssen

Physik. Institut der Universität,

Robert-Mayer-Strasse 2-4

D-6 Frankfurt am Main 1

T. (611) 7982345

A. Milojevic

Institute of Physics

Studentski trg $12 \mathrm{~N}$

POB 57

YU-11000 Belgrade

T. (11) 637569

P. Radvanyi

Institut de Physique Nucléaire

Faculté des Sciences, POB 1

F-91405 Orsay

T. (1) 9417185

Z. Wilhelmi

University of Warsaw, Institute

of Experimental Physics, Hoza 69

PL-00681 Warsaw

T. 216727

French Physical Society (3255) 4

Dels. M. Barrere

$$
\begin{aligned}
& \text { B. Dreyfus } \\
& \text { P. Radvanyi } \\
& \text { E. Schatzma }
\end{aligned}
$$

Pres. B. Dreyfus

Dépt. de Recherche Fondamentale

CEN/G - POB $85 X$

F-38041 Grenoble Cedex

Sec-Gen. P. Radvanyi

Société Française de Physique

Rue Croulebarbe 33

F-75013 Paris

T. (1) 7073298

German Physical Society(6020) 5

Dels. K.H. Hausser

W. Martienssen

E. Matthias

H.J. Queisser

A. Scharmann

Pres. H. Welker

Siemens AG

Forschungslaboratorium

Günther-Scharowsky-Strasse 2

D-852 Erlangen

Exec-Sec. W. Heinicke

Deutsche Physikalische Gesellschaft

Hauptstrasse 5

D-5340 Bad Honnef

T. (2224) 71061

Hellenic Physical Society (150) 1

Del. D. Sotiriou

Pres. G. Philokyprou

Sec-Gen. D. Marinos

Griveon Street 6

Athens $144 \mathrm{GR}$

T. (3021) 63570
Institute of Physics (15000) 5

Dels. L. Cohen K. Hoselitz

B.J. Mason

R.S. Pease

L.A.A. Thomas

Pres. B.J. Mason

Meteorological Office

Bracknell, Berks, UK

Exec-Sec. L. Cohen

The Institute of Physics

47 Belgrave Square

London SW $1 \times 80 \times$ UK

T. (441) $2356111 \mathrm{Tx} .918453$

Institute Ruder Boskovic (100)

Dir-Gen. V. Kundic

Bijenicka 54, POB 1016

YU-41001 Zagreb

T. (41) 424093

Israel Physical Society (240) 1

Del. C.G. Kuper

Pres. C.G. Kuper

Sec. R. Weil

Physics Department, Technion

Haifa Israel

T. (4) 225111

Italian Physical Society (1016) 3

Dels. G. Preparata

$$
\begin{aligned}
& \text { R.A. Ricci } \\
& \text { A. Zichichi }
\end{aligned}
$$

Pres. C. Castagnoli

Laboratorio di Cosmo-Geofisica

Corso Fiume 4

I-10133 Turin

Sec-Gen. G.A. Wolzak

Società Italiana di Fisica

Via L. degli Andalò 2

I-40124 Bologna
T. (51) 331554 
Netherlands' Physical Society

(2851) 4

Dels. J.J. Broeder

W.F. Druyvesteyn

A.M. Hoogenboom

P.R. Wyder

Pres. P.R. Wyder

Sec. G. van Middelkoop

Fysisch Laboratorium

Princetonplein 5

Utrecht NL

T. (30) 532517
Physical Sect. Union of Yugoslav Societies of Mathematicians, Physicists and Astronomers (200) Del. A. Milojevic

Pres. D. Ivanovic

Faculty of Electrotechnics

Bulevar Revolucije 73

YU-11000 Belgrade

Sec. J. Janjic

Institute of Physics, PMF

Dr. Ilije Duricica 4

YU-21000 Novi Sad

T. (21) 55622
Norwegian Physical Society (608) 2 Dels. T. Engeland I. Svare

Pres. T. Riste

Sec. G. Jarret

Institutt for Atomenergi, POB 40

$\mathrm{N}-2007$ Kjeller

T. (2) 712560 Tx. 16361 atom n
Physical Society of the German Democratic Republic (1299) 3 Dels. H. Guendel A. Lösche

Pres. R. Rompe

Sci-Sec. R. Linke

Physikalische Gesellschaft de

Deutschen Demokratischen Republik Am Kupfergraben 7

DDR-108 Berlin

T. (2) 200069
Physics Sect. Union of Czechoslovak Mathematicians and Physicists

(500) 2

Dels. J. Kaczer

S. Usacev

Pres. J. Kaczer

Sec. J. Fousek

Czechoslovak Academy of Sciences

$\mathrm{Na}$ Slovana 2

CS-18040 Prague-Liben

T. (422) 842241
Polish Physical Society (1600) 3 Dels. P. Decowski

$$
\begin{aligned}
& \text { Z. Wilhelmi } \\
& \text { J. Zakrzewski }
\end{aligned}
$$

Pres, Z. Wilhelmi

Sec-Gen. P. Decowski

University of Warsaw

Institute of Experimental Physics

Hoza, 69

PL-00681 Warsaw

T. 283031
Portuguese Physical Society (200) 1 Del. J.M. Gaspar Caraça

Pres. J. da Providencia

Departamento de Fisica

Faculdade de Ciências

Tecnologia, Univ. de Coimbra

Coimbra $\mathrm{P}$

Sec-Gen. H. Machado Jorge

Sociedade Portuguesa de Fisica

Av. da República, 37-4

Lisbon $1 \mathrm{P}$

T. (19) $671042 / 44$

Romanian Nat. Com. for Physics

(300) 1

Pres. I. Ursu

Sec. A. Corciovei

Inst. for Atomic Physics POB 5206

Bucharest R

T. 807040 Tx. 11397 fiatom r

Royal Irish Academy (55) 1

Del. B. Henderson

Pres. E.F. Fahy

Sec. E. Lalor

Irish National Committee for Physics

19 Dawson Street

Dublin 2 IRE

T. (31) 762570

Spanish Royal Society of Physics and Chemistry (200)

Del. J. Aguilar Peris

Pres. J.M. Gamboa

Inst ${ }^{\circ}$ de Quimica Fisica "Rocasolano"

CSIC. Serrano 119

Madrid-3

Sec-Gen. A. Tiemblo

Real Sociedad Espanola de Fisica

y Quimica

Cuidad Universitaria

Madrid-3 E

T. (1) 2433879
Swedish Physical Society (624) 2 Dels. C. Nordling

Pres. L. Minnhagen

Sec. W. Persson

Department of Physics

Lund Institute of Technology

POB 72

-220 07 Lund

T. (46) 124600

Swiss Physical Society (1200) 3

Dels. Ph. Choquard

$$
\text { M. Guenin }
$$

I. Zschokke-Gränacher

Pres. P. Choquard

Laboratoire de Physique Théorique

14 av. de I'Eglise Anglaise

$\mathrm{CH}-1006$ Lausanne

Sec. P. Erdös

Société Suisse de Physique

BSP Université de Lausanne

$\mathrm{CH}-1015$ Lausanne

T. (21) 240046 Tx. UNIVD 25110

Turkish Physical Society (282) 1 Del. A. Isin

Pres. E. Inönü

Bogaziçi Üniversitesi, PK 2, Bebek

Istanbul, TR

Sec. A. Ferendeci

CNAEM, PK 1, Havaalani

Istanbul TR

T. 737515

\section{Associate Members}

Battelle Geneva Research Centre, Carouge-Geneva, Switzerland

Brown, Boveri \& Company, Ltd.

Baden, Switzerland

Centre d'Etude de l'Energie Nucléaire, CEN/SCK, Mol, Belgium

CERN - European Organization for Nucl. Res. - Geneva, Switzerland Comitato Nazionale per I'Energia Nucleare, Frascati, Italy

Commissariat à I'Energie Atomique, Paris, France

Consiglio Nazionale delle Ricerche Rome, Italy

Culham Laboratory, Abingdon, UK

Danfysik A/S, Roskilde, Denmark

Deutsches Elektronen-Synchrotron

Hamburg, Fed. Rep. of Germany
European Space Agency,

Paris, France

Fiat SpA, Turin, Italy

Foundation for Fundamental Res. on Matter - FOM, Utrecht, NL

Hahn-Meitner-Institut, Berlin, FRG

IBM Europe, Paris, France

IBM Research Laboratory, Rüschlikon, Switzerland

Institut Max von Laue-Paul Langevin Grenoble, France Rome, Italy

Masson Editeurs, Paris, France

Max-Planck-Institut für Festkörper-

forschung, Stuttgart, FRG

Netherlands Energy Research

Foundation, Petten, The Netherlands
Istituto Nazionale di Fisica Nucleare,

North-Holland Publishing Co., Amsterdam. The Netherlands Osterreichische Studiengesellschaft für Atomenergie $\mathrm{GmbH}$, Vienna, A

N.V. Philips' Gloeilampenfabrieken, Eindhoven, The Netherlands

Physikalisch-Technische Bundesanstalt, Braunschweig, FRG

Laboratories RCA Ltd, Zürich, Switzerland

Research Institute of the Swedish National Defence, Stockholm, S

Siemens Aktiengesellschaft Berlin and Munich, Fed. Rep. of Germany Swiss Institute for Nuclear Research - SIN - Villigen, Switzerland

Taylor and Francis Ltd, London, UK

\section{EPS Advisory Committees}

\section{Applied Physics and Physics in Industry}

Ch. J.P. Hurault

Laboratoire d'Electronique et de

Physique Appliquée

3. Ave Descartes, POB 15

F-94450 Limeil-Brévannes

T. (1) 5699610

Sec. F.A. Kuipers

N.V. Philips' Gloeilampenfabrieken

Research Laboratories

Eindhoven, NL

T. (40) 742240

Zh.ll Alferov, A.F. loffe, PhysicoTechnical Institute, Leningrad

J.L. Delcroix, University Paris-Sud, Orsay

K. Differt, University, Stuttgart

A. Ferro-Milone, Nat. Electro-

technical Inst., Turin

K. Hoselitz, Sussex University, Brighton
H. Machado Jorge, Portuguese Physical Society, Lisbon

E. Nagy, Eötvös Lorand University, Budapest

C.P. O'Toole, Inst. for Industrial Research and Standards, Dublin

I. Purica, Institute for Atomic Physics, Bucharest

G. Raoult, Lab. de Radioélectricité \& Théorie du Solide, Aubière, F

T. Roettgers, Standard Elektrik Lorenz AG, Stuttgart

S. Shtrikman, Weizmann Institute, Rehovot

A. Sokollu, Marmara Research Institute, Istanbul

Z. Strugalski, Technical University, Warsaw

V. Valkovic, Institute Rudjer Boskovic, Zagreb

B.P. Veltman, University of Technology, Delft

\section{Conference Committee}

Ch. W.J. Merz

Sec. I. Eisenegger

RCA Laboratories Ltd

Badenerstrasse 569

$\mathrm{CH}-8048$ Zurich

T. (1) 526350

O. Akyuz, Bogaziçi Univ. Istanbul

M. Bertolotti, University, Rom

C.C. Butler, University of Technology, Loughborough, UK

J.M.G. Caraça, Portuguese Physical Soc., Lisbon

Ph. Catillon, CEA Saclay, Gif-sur-Yvette

C. Cesarsky, CEA Saclay, Gif-sur-Yvette

A. Doenszelman, Zeeman Lab. Amsterdam

I.A. Dorobantu, Institute for Atomic Physics; Bucharest

M. Fitaire, University Paris XI, Orsay

M. Guenin, University, Geneva

P.G. Hansen, CERN, Geneva

J.P. Hurault, Lab. of Elec. and App. Phys., Limeil-Brévannes Sciences, Warsaw
A. Kujawski, Polish Academy of

\section{I.O.M. Delegates}

Delegates are elected by a ballot of all 


\section{Physics Education}

Ch. A. Janner

Institute for Theoretical Physics

Nijmegen University, Toernooiveld

Nijmegen NL

T. (80) 558833

Sec. M.G. Ebison

Institute of Physics

47 Belgrave Square

London SW1X 8QX UK

T. (1) 2356111

M. Anastassiadis, Athens

E.R. Andrew, University, Nottingham

G. Aubert, CNRS, Grenoble

J. Auth, DDR NationalCommmittee for

Physics, Berlin

G. Born, Gesamthochschule, Duisburg

D. Brinkmann, University, Zurich

M. Chytilova, Brno

M. Dede, University, Debrecen

B. Eman, Institute Rudjer Boskovic,

Zagreb

B. Karczewski, University, Warsaw

K.J. Knutsen, Trondheim

H.G. Kramers, Kamerlingh Onnes

Laboratory, Leiden

W. Kuhn, University, Giessen

H. Machado Jorge, Portuguese Physica Society, Lisbon

V. Meisalo, University, Helsinki

E. Mendoza, Hebrew University, Jerusalem

R. Nasuhoglu, Faculty of Science, Ankara

A. Raev, Faculty of Physics, Sofia

S. Rapeanu, Institute for Atomic

Physics, Bucharest

L. Samuelsson, University, Linköping

J. Vlachy, Prague

Representatives of Unesco:

N. Joel, Paris

B. Robinson, Paris

The Committee publishes a newsletter entitled Europhysics Education News which is sent free of charge to a limited number of interested readers.

\section{Europhysics News}

Ch. J. Muller*

Université de Genève, Section de

Physique, boulevard d'Yvoy 32

$\mathrm{CH}-1211$ Geneva 4

T. (22) 219355

Sec. E.N. Shaw

Editor, EPS, POB 69

$\mathrm{CH}-1213$ Petit-Lancy 2

T. (22) 931130

J. Auth, DDR National Committee for Physics, Berlin

*G.J. Béné, University, Geneva

A.B. Berezin, A-F loffe Physico-

Technical Institute, Leningrad

M. Bloch, CEN Saclay, Gif-sur-Yvette

E. Burzo, Institute for Atomic

Physics, Bucharest

I. Butterworth, Imperial College of Science and Technology, London

J.M.G. Caraça, Portuguese Physical Society, Lisbon

L. Cohen, Inst. of Physics, London

L. Colombo, Institute Rudjer Boskovic, Zagreb

A. Doenszelmann, Zeeman Laboratory, Amsterdam

F. Engelmann, FOM, Jutphaas, NL

P. Erdös, University, Lausanne

S. Focardi, Institute of Physics, Bologna

A.D. Fokker, Astronomical Institute, Utrecht

M. Guenin, University, Geneva

E. Gültekin, CNAEM, Istanbul

K. Kromphardt, Redaktion Physikalische Blätter, Bad Honnef

D.J. Kroon, N.V. Philips' Gloeilampenfabrieken, Eindhoven

B. Lalovic, Institute Boris Kidric Belgrade

A.R. Mackintosh, University, Copenhagen

*G.R. Macleod, CERN, Geneva

G. Marx, Eötvös Lorand University, Budapest

F. Netter, CEN Saclay, Gif-sur-Yvette

'W.S. Newman, CERN, Geneva

*D. Pohl, IBM Laboratory, Rüschlikon

*J.A. Schwarzmüller, Battelle Institute,

Geneva

A. Strzalkowski, University, Cracow
G. Thomas, EPS, Secretariat

J.P. Toennies, Max-Plank-Institut,

Göttingen

$H$. van Krugten, University, Delft

* = Member of Editorial Board

${ }^{\circ}=$ Meetings Compilation

\section{Physics and Society}

Ch. L.J.F. Broe

Technical University

POB 513

Eindhoven NL

T. (40) 479111

Sec. D. Schumacher

IHK Rhein-Neckar

Hans-Böcker-Strasse 4

D-6900 Heidelberg

T. (6221) 23999

S. Aegerter, Fond. Nat. Suisse Berne

G.J. Béné, University, Geneva

D. Berényi, Hungarian Academy of Sciences, Debrecen

I. Brandus, State Committee for

Nuclear Research, Bucharest

J.M.G. Caraça. Portuguese

Physical Society, Lisbon

E.T. Ferguson, N.V. Philips'

Gloeilampenfabrieken, Eindhoven

M.J. Higatsberger, University, Vienna

S. Pallua, Rudjer Boskovic

Institute, Zagreb

D.H. Parkinson, Min. of Defence, London

G.A. Smolensky, Academy of Sciences, Leningrad

G. Toraldo di Francia, University, Florence

T. Türkeli, Faculty of Science, Istanbul

H.G. van Bueren, Astronomical Institute, Utrecht

H. van Regemorter, Paris Observatory, Meudon

J. Vlachy, Prague

W. Zych, University, Warsaw

\section{Publications}

R. Dobbs

Bedford College

Regents Park

ondon NW1 4 NS UK

T. (1) 4864400

Sec. P.F.A. Klinkenberg

Zeeman Laboratorium,

Plantage Muidergracht 4

1004 Amsterdam, NL

T. (20) 5229111

E. Aktulta, Faculty of Science,

Istanbul

D. Barb, Institute for Atomic Physics Bucharest

G. Bellini, Inst. of

Physical Sciences, Milan

A.B. Berezin, A-F loffe Physico-

Technical Institute, Leningrad

M. Bertolotti, University, Rome

A. Bialas, Jagellonian University,

Cracow

J. Campos, University, Madrid

J. de Boer, University, Amsterdam

J. Des Cloizeaux, CEN Saclay,

Gif-sur-Yvette

J.P. Dougherty, University, Cambridge

B. Giovannini, University, Geneva

M. Guenin, University, Geneva

J. Krempasky, Slovak Techn. Univ.

Bratislava

H. Machado Jorge, Portuguese Physica Society, Lisbon

P.F. Meier, University, Zurich

A. Moljk, Faculty for Natural Sciences

and Technology, Ljubljana

G. Nier, Redaktion «Physikalische Berichte", Braunschweig

H.A. Olsen, University, Trondheim

C.I. Pedersen, Inst. of Physics, Bristol

G. Preparata, CERN, Geneva

G. Raoult, Lab. de Radioélectricité et Théorie du Solide, Aubière F

G. Turchanyi, University, Budapest

P. Urban, University, Graz

N. Urli, Inst. Ruder Boskovic, Zagreb

C. van der Leun, University, Utrecht

L. van Gerven, University, Leuven

J.-Ch. Vienot, University, Besançon

A. Wroblewski, University, Warsaw

\section{EPS Divisions}

\section{Astronomy and Astrophysics Solar Section (68}

\section{Division (338)}

\section{Ch. K. Pinkau}

Max-Planck-Institut für Physik und

Astrophysik, Institut für

extraterrestrische Physik

D-8046 Garching near Munich

T. (89) 3299518

Sec. C. Cesarsky

SEP-CEN Saclay, BP 2

F-91405 Gif-sur-Yvette

T. (1) 9418000

Ch. C. Jordan

Department of Theoretical Physics

University of Oxford

12 Parks Road

Oxford OX1 3PJ UK

T. (865) 59291

Sec. A.D. Fokke

Sterrekundig Instituut te Utrech

Sterrewacht "Sonnenborgh")

Zonnenburg 2

3512 Utrecht NL

T. (30) 312841

Board Members:

R.M. Bonnet, Lab. de Phys. Stell. et Plan., Verrières le Buisson

C. Chiuderi, Observatory, Florence

M. Kuperus, Astronomical Institute, Utrecht

P. Maltby, Inst. of Theor. Astrophysics, Oslo

H. Nussbaumer, ETH, Zurich

J.-C. Pecker, Institute of Astrophysics, Paris

M. Pick, Observatory, Meudon

I.W. Roxburgh, Queen Mary College,

London

J.O. Stenflo, Astronomical Institute, Lund

C. Zwaan, Astronomical Institute, Utrecht

\section{Atomic Physics Division (526)}

Ch. A.H. Gabriel

ARD, Culham Laboratory

Abingdon OX14 3DB UK

T. (235) 21840

Sec. I. Martinson

Fysiska Institutionen

Lunds Universitet

Sölvegatan 14

S-223 62 Lund

T. (46) 124620

New board elections will be held in the Summer, 1978

\section{Atomic Spectroscopy Sect.}

(EGAS) (327)

Ch. D.N. Stacey

Clarendon Laboratory, Parks Road

Oxford OX13PU UK

T. (865) 59291

Sec. A. Dönszelmann

Zeeman Laboratorium

Plantage Muidergracht 4

1004 Amsterdam NL

T. (20) 5222095

Board Members:

T. Andersen, University, Aarhus

J. Bauche, CNRS II, Orsay

B. Cagnac, ENS, Paris

W.R.S. Garton, Imperial Coll., London

H. Hühnermann, University, Marburg

M.D. Kunisz, Jagellonian University,

$$
\text { Cracow }
$$

J.C. Lehmann, University VI, Paris

I. Martinson, University, Lund

R. Winkler, T.U., Berlin-West

Board Members:

The Chairmen and Secretaries of the Sections are also members of the Divisional Board

R. Damburg, Latvian Academy of 


\section{lectronic and Atomic}

Collisions Section (338)

\section{Ch. F. Linder}

Universität Kaiserslautern

Pfaffenbergstrasse

D-6750 Kaiserslautern

T. (631) 8542383

\section{Sec. F.H. Read}

Department of Physics

Schuster Laboratory, The University

Manchester M13 9PL UK

T. (61) 2734873

Board Members:

V.V. Afrosimov, Academy of Sciences, Leningrad

B. Cobic, Boris Kidric Inst.

of Nucl. Sci., Belgrade

P. Dahl, University, Aarhus

F.A. Gianturco, Chemical-Physics

Institute, Bari

H.G.M. Heideman, University, Utrecht

Ch. Joachain, Free Univ., Brussels

T.D. Märk, Atomic Phys. Inst.,

Innsbruck

V. Martisovits, Comenius Univ.,

Bratislava

\section{Condensed Matter \\ Division (853)}

Ch. M. Balkansk

Sec. M. Voos

Pierre et Marie Curie Univ.

4, Place Jussieu

F-75230 Paris Cedex 05

T. (1) 3297786

Board Members

P. Averbuch, Scientific and Medical Univ., Grenoble

G. Bäckström, University, Umea B. Giovannini, University, Geneva O.S. Heavens, University, York

W.J. Huiskamp, Kamerlingh Onnes Laboratory, Leiden

Low Temperature Section (254) Ch. W.J. Huiskamp

Kamerlingh Onnes Laboratory

Rijksuniversiteit te Leiden

Nieuwsteeg 18

Leiden, NL

T. (71) 141341

Sec. J. Souletie

Centre de Recherches sur les

Très Basses Températures

25, Av. des Martyrs, POB 166

F-38042 Grenoble Cedex

T. (76) 969837

Committee Members:

G. Eilenberger, Solid State Inst., Jülich

T. Lindqvist, University, Uppsala

C. Rizzuto, University, Genoa

R.S. Safrata, Czechoslovak Academy of Sciences, Prague

Y.V. Sharvin, Institute for

Physical Problems, Moscow

\section{Magnetism Section (352)}

Ch. E.P. Wohlfarth

Imperial College, Queens Gate

London SW7 2BZ, UK

T. (1) 5895111

Committee Members

A.S. Borovik-Romanov, Institute

for Physical Problems, Moscow

G. Busch, ETH, Zurich

P. Erdös, University, Lausanne

W. Haubenreisser, Institute for

Magnetic Materials, Jena

A. Hubert, University, Erlangen

V. Kambersky, Academy of Sciences, Prague

P. Mazzetti, Galileo Ferraris Institute, Turin

A.R. Miedema, Philips Research Laboratories, Eindhoven

L. Pal, Central Physics Research Institute, Budapest

R. Pauthenet, National Intense Field Service, Grenoble

S. Szczeniowski, University, Warsaw

L. van Gerven, University, Leuven

\section{Molecular Physics Section}

340)

Ch. I. Kovacs

Department of Atomic Physics, Polytechnical University, Budafoki ut 8 , Budapest $1521 \mathrm{H}$

Budapest 152

Sec. F. Comes

Institut für Physikalische Chemie

der Universität.

Robert-Mayer-Strasse 11

D-6 Frankfurt/Main 1

Board Members :

G. Amat, University Paris XI, Orsay

R.F. Barrow, University, Oxford

P.K. Carroll, University, Dublin

J. Depireux, Liège Univ., Sart Tilman

K. Dressler, ETH, Zurich

B. Jezowska-Trzebiatowska,

University, Wroclaw

A. Lagerqvist, University, Stockholm

S. Leach, CNRS, Orsay

A. Personov, Academy of Sciences Moscow

C. Hilsum, Royal Signals and Radar Establishment, Malvern, UK

Yu. Ossipyan, Solid State Physics

Institute, Moscow

T. Roettgers, Standard Elektrik

Lorenz, Stuttgart

G. Szigeti, Hungarian Academy of Sciences, Budapest

C.J. Todd, P.O. Research Centre,

Martlesham Heath, Ipswich

L.G. van Gerven, University of

Leuven, Leuven

I.M. Ward, University, Leeds

E.P. Wohlfarth, Imperial College London

Macromolecular Physics Section (165)

Ch. I.M. Ward

Department of Physics

University of Leeds

Leeds LS2 9JT, UK

T. (532) 31751

Committee Members :

A.L. Buchachenko, Institute of Chemical Physics, Moscow

J.C. Fatou, Plastics and Rubber Institute, Madrid

E.W. Fischer, University, Mainz

W. Holzmüller, Karl Marx University, Leipzig

H.H. Kausch, EPFL, Lausanne

A.J. Kovacs, Macromolecular Research Centre, Strasbourg

J. Kubat, University of

Technology, Göteborg

J.P. Mercier, High Polymer Laboratory, Louvain-la-Neuve

A.J. Pennings, Rijks University, Groningen

G. Zerbi, CNR, Milan

A. Ziabicki, Polish Academy of Sciences, Warsaw

\section{Metals Section (389)}

Ch. P. Averbuch

Université Scientifique

et Médicale de Grenoble,

POB 53

F-38041 Grenoble Cedex

T. (76) 548152

Committee Members

G. Caglioti, Inst. of Nuclear Engineering, Milan

R.M.J. Cotteril, Technical University, Lyngby

D.J. Fabian, Strathclyde University, Glasgow,

J. Muller, University, Geneva

W. Schilling, Kernforschungsanlage Jülich
Semiconductors and

Insulators Section (463)

Ch. C. Hilsum

Royal Signals and Radar Establishment

St. Andrew Road

Gt. Malvern, Worcs WR14 3PS, UK

T. $(6845) 2733$

Committee Members:

F.W. Ainger, Plessey Co., Towcester

Zh. I. Alferov, A.F. loffe, Physico-

Technical Institute, Leningrad

G. Chiarotti, University, Rome

P. Grosse, Technische Hochschule, Aachen

\section{Computational Physics}

Group (334)

Ch. P. Burke

Sec. K.A. Berrington

The Queen's University of Belfast

Belfast BT7 1 NN Northern Ireland

Tel. 45133

Board Members:

D. Biskamp, Max-Planck Inst. of

Plasma Physics, Garching

M. Bloch, CEN-Saclay, Gif-sur-Yvette

S. Cuperman, University, Tel-Aviv

G. Diercksen, Max-Planck Inst. of

Physics and Astrophysics, Munich

F. James, CERN, Geneva

E.W. Laing, University, Glasgow

Nadrchal, Inst. of Solid State

Physics, Prague

R. Zelazny, Inst. of Nuclear

Research, Swierk

\section{Nuclear Physics Division} (547)

Ch. C. van der Leun

Fysisch Laboratorium, Sorbonnelaan 4 , Utrecht NL

T. (30) 532513

Sec. J. Sharpey-Schafer

University of Liverpool, POB 147

Liverpool L69 3 BX UK

T. (51) 86559911

Board Members :

K. Alder, Institute for Physics, Basle

D. Berenyi, Institute of

Nuclear Research, Debrecen

K. Bethge, Institute for Nuclear Physics,

Frankfurt/Main

N. Cindro, Institute Ruder Boskovic,

Zagreb

E. Cotton, CEN Saclay, Gif-sur-

Yvette

P.G. Hansen, CERN, Geneva

M. Ivascu, Institute for Atomic

Physics, Bucharest

T. Mayer-Kuckuk, University, Bonn

S.G. Nilsson, Institute of Technology, Lund

R. Ricci, National Laboratory, Padua

V. Soloviev, Joint Institute for Nuclear

Research, Moscow

A. Strzalkowski, Jagellonian University, Cracow

I. Talmi, Weizmann Institute of

Sciences, Rehovot

G. Vouvopoulos, NRC Demokritos,

Attiki

\section{Quantum Electronics Division (257)}

Ch. S.D. Smith

Heriot Watt University

Riccarton, Currie

Edinburgh $\mathrm{EH} 144$ AS

$\mathrm{T}$ (31) 4495111

Sec. R. Dändliker

Brown-Boveri Research Centre

$\mathrm{CH}-5405$ Baden-Dättwil

T. (56) 848411

Surface and Interface

Section (181)

Ch. C.J. Todd

Post Office Research Centre

Martlesham Heath

Ipswich IP5 7RE, UK

T. (473) 643287

Committee Members

G. Ertle, University, Munich

B. Feuerbacher, ESA, Noordwijk

R. Kern, Scientific Centre of

Saint-Jérôme, Marseille

H. van Hove, Catholic University, Leuven

A. van Oostrom, Philips Research

Laboratories, Eindhoven

\section{High Energy and Particle}

Physics Division (477)

Ch. G. Preparata

CERN, TH Division

$\mathrm{CH}-1211$ Geneva 23

T. (22) 832801

Sec. I. Butterworth

Physics Dep., Imperial College

Prince Consort Road

London SW7 2AZ UK 
29 Jan.-2 Feb.

Int. Symposium on Thermodynamics of Nuclea Materials

Conference Service, IAEA, POB 590, A-1011 Vienna 30 Jan.-1 Feb.

Future Energy Concepts

London, UK

IEE Conf. Dept., Savoy Place, London WC2R OBL

Ab: $1.5 .78 / P: 31.8 .78$

5-9 March

Zurich, Switzerland

2nd Int. Conf, on Infrared Physics (CIRP 2)

CIRP 2, c/o F. Kneubühl, Solid State Physics

Laboratory, ETH Hönggerberg, $\mathrm{CH}-8093$ Zurich

12-14 March San Francisco, USA

Particle Accelerator Conference

Technical Activities Board, IEEE, 345 E. 47th Street, New York, NY 10017

12-17 March Berne, Switzerland

Albert Einstein Centenary Celebration

A. Mercier, Institut für Theoretische Physik, Sidlerstrasse 5, CH-3012 Bern

26-29 March Chicago, USA General Meeting of American Physical Society American Physical Society, 335 E. 45th Street New York, NY 10017

Ab: 5.1 .79

\section{6-29 March}

Cambridge, UK

2nd European Surface Science Conf. (ECOSS2)

Interdisciplinary Surface Science Conf. (ISSC4)

P.M. Williams, V.G. Scientific Ltd., Imberhorne Lane, East Grinstead, Sussex, RH19 10Y

A: 1.2 .79 / PP: $1.12 .78 / 350$

27-29 March

Sheffield, UK

Trends in On-Line Computer Control Systems

IEE Conf. Dept., Savoy Place, London WC2R OBL Ab: $12.6 .78 / P: 6.11 .78$

28-30 March

Oxford UK

2nd Conf. on Mechanical Properties of Materials at High Rates of Strain

The Meetings Officer, The Institute of Physics, 47 Belgrave Square, London SW $1 \times 80 X$ Ab: 1.9 .78

\section{8-30 March}

Birmingham, UK

2nd National Reliability Conf.: Reliability - The Key to Survival

National Centre of Systems Reliability, Wigshaw Lane, Culcheth, Warrington WA3 4NE

Ab: $30.7 .78 / P: 30.9 .78$

28-30 March

Birmingham, UK

Nuclear Structure and Elementary Particle Physics The Meetings Officer, The Institute of Physics 47 Belgrave Square, London SW 1 X $80 X$

2-5 April

Cambridge, UK

4th Int Conf, on Deformation, Yield and Fracture of Polymers

J.N. Ratcliffe, The Plastics and Rubber Institute 11 Hobart Place, London SW1W OHL

Ab: 1.8 .78

2-6 April

San Diego, CA, USA

6th Int Vacuum Metallurgy Conf. on Special Meltin and Metallurgical Coatings

R.W. Buckman, Jr. POB 18006

Pittsburgh, PA 15236

Ab: 10.9.78/PP

3-5 April

Nottingham, UK

2nd Int. Conf. on Low Light and Thermal Imaging Suzanne J. Hall, Conference Secretary, IEE, Savo Place, London WC2R OB

A: $3.4 .79 / \mathrm{Ab}: 1.11 .78 / \mathrm{PP} / 500$

3-6 April

Nijmegen, The Netherlands

SEAS Spring Technical Meeting (Computer Graphic Applications)

Share European Association. Toernooiveld Nijmegen, NL

A: $1.3 .79 / \mathrm{PP} / 200 / \mathrm{hFl}$. 425.-

\section{4-6 April}

Bath, UK

Strength and Structure in Carbons and Graphites The Meetings Officer, The Institute of Physics, 47 Belgrave Square, London SW $1 \times 80 X$

Ab: 30.11 .78

4-6 April

Spectroscopy at Millimetre and Sub-millimetre Wavelengths using Coherent Sources

D.C. Lainé, Department of Physics, University of Keele Staffordshire ST5 $5 \mathrm{BC}$

A: 7.2.79/Ab: 7.2.79/NP / 100

8-11 April Lake Yamanaka, Japan 5th Int. Conf. on Positron Annihilation (ICPA-5 K. Fujiwara, Conf. Secretary, Inst. of Physics, College of General Education, Univ. of Tokyo, Komaba 3-8-1, Tokyo 153

\section{9-11 April}

Birmingham, UK

Y.A. State Seled Species

Y.A. Fish, The Chemical
House, London W1V OBN

A: $12.3 .79 / A b: 1.5 .78 / P: 1.12 .78 / 150$

9-12 April Enschede, NL

2nd Int. Symposium: Incoherent Light Sources

E.W. ten Napel, Dept. of Applied Physics,

Twente University of Technology,

POB 217, Enschede

A: Feb. 79 / Áb: 1.6.78 / P: Nov. 78 / $100 /$

hFI. 200. -

9-12 April

11 th Nat. Atomic and Molecular Physics Conf

The Meetings Officer, The Institute of Physics,

47 Belgrave Square, London SW $1 \times 80 X$

10-12 April

Oxford, UK

Microscopy of Electronic Materials

The Administrator, Royal Microscopical Society, 37/38 St. Clements, Oxford OX4 1AJ

\section{7-20 April}

Oxford, UK

Electrostatics 1979

The Meetings Officer, The Institute of Physics, 47 Belgrave Square, London SW $1 \times 80 X$

Ab: 15.12 .78 / PP: 28.3.79

22-27 April

Boulder, CO, USA

Workshop on Solar-Terrestrial Predictions

R.F. Donnelly, STP / P-W Program, Space

Environment Lab., NOAA ERL,

Boulder, CO 80302

Ab: 1.11 .78

23-26 April

Washington, DC, USA

General Meeting of American Physical Society The American Physical Society, 335 E. 45th Street, New York, NY 10017

$A b: 2.2 .79$

23-28 April Jablonna nr. Warsaw, PL

9th Europhysics Conf. on Macromolecular Physics: Structure and Properties of Polymer Networks

A. Ziabicki, Institute of Fundamental Technological Research, Polish Academy of Sciences, Swietokrzyska 21, PL 00-049 Warsaw

A: 31.1 .79 / Ab: 30.10 .78 / PP / 120 English / IUPAC

\section{April-2 May}

International Microwave Symposium

Orlando, FL, USA

College of Engineering. University of

South Florida, Tampa, FL 33620

May

Jülich, FRG

Int. Symposium on Physics and Chemistry of Fission Conference Service, IAEA, POB 590, A-1011 Vienna

May or June

Autrans, France

4th EENC: New NMR Techniques and Applications

P. Servoz-Gavin, CEA-CEN de Grenoble, B.P. 166 Centre de Tri, F-38042 Grenoble Cedex

7-11 May

Hamburg, FRG

2nd European Nuclear Conference (ENC 79)

Geschäftsstelle Kerntechnische Gesellschaft

Deutsches Atomforum e.V., Heussallee 10, D-5300 Bonn

8-10 May

San Francisco, CA, USA 24th National SAMPE Symposium and Exhibition Marge Smith, SAMPE, POB 613, Azusa, CA 91702 Ab: 1.8 .78

\section{4-18 May}

International Switching Symposium

Paris, France

Secrétariat, Colloque International de Commutation, 11 rue Hamelin, F-75783 Paris Cedex 16

Ab: May 78 / PP / English, French

16-17 May

London, UK

Ultra-Short Laser Pulses

The Royal Society, 6 Carlton House Terrace. London SW1Y 5AG

\section{쿠 17-23 May}

Gamma Ray Astronomy after COS-B

C. Cesarsky, DPh/EP/ES-Bât. 28, Centre d'Etudes Nucléaires de Saclay, B.P. No. 2, F-91190 Gif-sur-Yvette,

$$
\text { France }
$$

70 / inv. / US \$ 220.

21-23 May

Lyon-Villeurbanne, France Annual Conf.: Micro-analysis - Scanning of Electron Microscopy

Soc. Française de Microscopie Electronique, $\mathrm{PP}$

67 rue Maurice Günsbourg, F-94200 Ivry

\section{1-27 May}

Moscow, USSR

8th IMEKO Congress: Measurement for Progress in Science and Technology

IMEKO Secretariat, POB 457, H-1371 Budapest

Ab: 31.5.78 / P: 31.1.79/English, Russian

US $\$ 150$. - Rbl. 120 .

24-28 May

2nd "Lund" Int. Conference on Deep Levels in Semiconductors

A. Mircea, LEP, 3 ave. Descartes,

F-94450 Limeil-Brévannes

$80 /$ inv.

29 May-9 June

Bangalore, India

22nd COSPAR PLenary Meeting and Symposia

Z. Niemirowicz, COSPAR Secretariat, $51 \mathrm{Bd}$ de Montmorency, F-75016 Paris, France

PP / 400 / English, French / COSPAR

3-8 June

Atlanta, USA

Annual Meeting of American Nuclear Society

David G. Pettengill, American Nuclear Society, 555 North Kensington Ave

La Grange Park, IL 6052.5

4-6 June

Montreal, Quebec, Canada

International Conference on Plasma Science

Claude Richard, Hydro-Quebec, Research Institute, POB 1000, Varennes, 501 2PO, Quebec

11-15 June

Washington, DC, USA

Inter-Congress Symposium: Accuracy in Powder Diffraction

S. Block, Crystallography Section, National Bureau IUCr of Standards, Washington, DC 20234

11-16 June Keszthely, Lake Balaton, Hungary Large Amplitude Collective Nuclear Motions

A. Kiss, Institute for Atomic Phys., Eötvös Lorand University, Puskin u. 5-7, $\mathrm{H}-1088$ Budapest

A: 1.2.79/Ab: 1.3.79/P: 11.6.79/100/Sfr. 120.IOM, Sfr. 140. - others

12-16 June

Stuttgart, FRG

6 th Int. Conf. on Solid Compounds of Transition Elements

H. Schulz, Max-Planck-Institut für Festkörperforschung, РОВ 800665 .

D-7000 Stuttgart 80

A: Feb. $79 /$ P: 1.12.78 / 250

Sfr. 150. - approx

18-21 June Vancouver, BC, Canada

1979 Congress of the Canadian Association of Physicists

Canadian Association of Physicists,

Suite 903, 151 Slater Street,

Ottawa, Ont., Canada K1P $5 \mathrm{H} 3$

18-22 June Prague, Czechoslovakie

5. Internationaler Kongress für Reprografie

Sekretariat Komitetu pro pozvoj reprografie v CSSR $\mathrm{Na}$ Frantiski 32, CS-11015 Praha 1 - Stare Mesto

19-22 June

Neutrino 79

Bergen, Norway

Cecilia Jarlskog, Dept. of Physics, University of Bergen, N-5014 Bergen

PP / 200 / inv. / US \$ 50. — approx.

27 June-4 July Geneva, Switzerland 1979 EPS Int. Conf. on High Energy Physics

Secretariat, European Physical Society, P.O. Box 69 CH-1213 Petit-Lancy 2

PP / 600 / inv. 
2-7 July Lyons, France

1Oth Int. Conf. on Solid State Nuclear Track Detectors R. Schmitt, Université Lyon I, Rayonnement Cosmique 43 bd du 11 Novembre, F-69621 Villeurbanne Cedex

inv. / English, French

3-5 July

Univ. of Sussex, UK

Int. Conf.: Computer Aided Design and Manufacture of Electronic Components Circuits and Systems IEE Conf. Dept., Savoy Place, London WC2R OBL Ab: 6.10 .78 / P: 5.2 .79

3-5 July

Southampton, UK

Numerical Solution of Highly Nonlinear Problems: Symposium on Fixed Point Algorithms and Complementarity

W. Forster, University of Southampton, Faculty of Mathematical Studies, Southampton SO9 5NH

9-14 July Grenoble, France
14th Int. Conference on lonisation Phenomena in

Grenoble, France Gases (ICPIG 14)

C. Pomot, Comité d'Organisation ICPIG 14, Avenue d'Innsbruck, F-38029 Grenoble Cedex

A: 1.5.79 / Ab: 16.3.79 / PP / 800 / English, French, German, Russian / URSI / FF 500.-

17-20 July

New York, USA

Int. Magnetics and Magnetism and Magnetic Materials Conf. (INTERMAG and M3)

Paul Shumate, Bell Laboratories, Murray Hill, NJ 07974

$\mathrm{PP} / \mathrm{800}$

\section{July-3 Aug.}

Le Creusot, France

7 th International High Pressure Conference

B. Vodar, LIMHP-CNRS, F-93430 Villetaneuse Ab: 15.1 .79

August

San Diego, CA, USA

National Heat Transfer Conference

R. Viskanta, School of Mechanical Engineering Purdue University, West Lafayette,

IN 47907

5-10 Aug

Boston, MA, USA

Energy Conversion Engineering Conference

David G. Pettengill, American Nuclear Society, 555 North Kensington Avenue,

La Grange Park, IL 60525

6-10 Aug.

Lyngby, Denmark

IUTAM Symposium: Physics and Mechanics of Ice

P. Tryde, Institute of Hydrodynamics and Hydraulic Engineering, Technical University of Denmark Bldg. 115, DK-2800 Lyngby

A: 1.4 .79 / Ab: 1.4 .79 / P: $1.4 .79 / 50 /$ inv.

6-10 Aug. Mont Tremblant, Quebec, Canada IAU Symposium: Interstellar Molecules

J.L. Locke, Herzberg Institute of Astrophysics, National Research Council,

Ottawa, Canada K1A OR6

PP / 200 / inv. / English, French / IAU

7-10 Aug. Toronto, Canada

IAU Symposium: Close Binary Stars: Observation and Interpretation

C.T. Bolton, David Dunlap Observatory, Richmond Hill, Ont., Canada 14C 4Y6

PP / 150 / inv. / English, French / IAU

7-10 Aug.

College Park, MD, USA

IAU Symposium: Radio Physics of the Sun

M.R. Kundu, Astronomy Program, Univ. of Maryland, College Park, MD 20742

PP / $150 /$ inv. / IAU

12-18 Aug.

Oslo, Norway

8th Int. Mass Spectrometry Conference

O.H.J. Christie, Laboratory for Mass Spectrometry University of Oslo, P.B. 1048, Blindern, Oslo 3 IUPAC

13-17 Aug.

Copenhagen, Denmark 5th European Crystallographic Meeting (ECM-5) Ingrid Kjøller Larsen, Department of Chemistry BC, Royal Danish School of Pharmacy, Universitetsparken 2, DK-2100 Copenhagen

14-23 Aug. Montreal, Canada
17 th General Assembly of International Astronomica

14-23 Aug. Montreal, Canada
17 th General Assembly of International Astronomica Union

E.A. Müller, General Secretary, IAU, Observatoire de Genève, $\mathrm{CH}-1290$ Sauverny

19-24 Aug.

Jerusalem, Israe

12 th Int. Conf. on Medical and Biological Engineering

5 th Int. Conf. on Medical Physics

Conference Secretariat, POB 16271, Tel Aviv
19-25 Aug. Mont Tremblant, Quebec, Canada Sagamore VI: 6th Conf. on Charge, Spin and Momentum Densities

V.H. Smith, Department of Chemistry, Queen's University, Kingston, Ontario, Canada K7L 3N6 IUC

19-26 Aug.

10th Int. Radiocarbon Conference

Hans Oeschger, Physikalisches Institut, Universität Bern, Sidlerstrasse 5, CH-3012 Berne

20-24 Aug.

Cambridge, UK

3rd Int. Conf. on Mechanical Behaviour of Materials (ICM3)

Conference Secretariat, ICM 3, Engineering Dept. University of Cambridge, Trumpington Street, Cambridge CB2 1PZ

20-24 Aug.

Göttingen, FRG

7 th Int. Colloquium on Gasdynamics of Explosions and Reactive Systems

A.K. Oppenheim, Univ. of California,

Berkeley, CA 94720

Ab: 1.2 .79

21-24 Aug.

Cryogenic Engineering Conference

Dee Belsher, National Bureau of Standards

27-30 Aug.

London, Ontario, Canada

IAU Colloquium: Turbulence and Mass Loss in Stella Atmospheres

D.F. Gray, Dept. of Astronomy, University of

W. Ontario, London, Ontario, Canada N6A 5B9

PP / 100 / inv. / English, French / IAU

27-31 Aug.

Victoria, BC, Canada

Star Clusters

S. van den Bergh, Dominion Astrophysical Observatory, $5071 \mathrm{~W}$. Saanich Road, Victoria, B.C. V8X $3 \times 3$

PP / 120 / inv. / English, French / IAU

\section{7-31 Aug.}

Helsinki, Finland

27 th Congress of International Union of Pure and Applied Chemistry

J. Larinkari, Kemian Keskusliitto, POB 13028, IUPAC abianinkatu 7B, SF-00131 Helsinki 13

27 Aug.-1 Sept.

4 th Int. Symp. on Plasma Chemistry A: 15.9 .78 / PP / IUPAC

28-31 Aug.

Symposium on the Mechanics of Sound Generation in Flows

E.A. Müller, Max-Planck-Institut für Strömungsforschung, Böttingerstrasse $6 / 8$

-3400 Göttingen

inv. / ICA, IUTAM, AIAA

29-31 Aug.

Neutron Scattering and Magnetism

K.H. Fischer, Conf. Secr., KFA Jülich, IFF, Postfach 1913, D-5170 Jülich

PP / 120 / DM 60. - approx.

29 Aug.-4 Sept.

Kyoto, Japan

11 th Int. Conf. on Physics of Electronic and Atomic Collisions

T. Takayanagi, Inst. for Space and Aeronautica Sciences, Komaba 4-6-1, Meguro-ku, Tokyo 153

Ab: 20.4 .79

September

Frankfurt/Main, FRG

14th European Congress on Molecular Spectroscopy

F.J. Comes, Inst. f. Physikalische Chemie der

Universität, Robert-Mayerstrasse 11 600 D-6 Frankfurt/Main 1

2-10 Sept. Davos, Switzerland
30th General Assembly of International Union of Pure

Davos, Switzerland and Applied Chemistry

IUPAC Secretariat, 2/3 Pound Way, Cowley Centre, Oxford OX4 3YF, UK

3-7 Sept.

Ljubljana, Yugoslavis

4th European Conf. on Ferroelectricity (EMF-4)

R. Blinc, Institute 'Josef Stefan', Jamova 39 POB 199/IV, YU-61001 Ljubljan

A: 1.6.79/Ab: 1.3.79/P: 1.6.79/300/ US \$ 80 .

4-7 Sept.

Edinburgh, UK Workshop on Electron Microscopy Preparation Techniques

The Administrator, Royal Microscopical Society, 37/38 St. Clements, Oxford OX4 1AJ

10-13 Sept.

Birmingham, UK

3rd Int. Conf.: Dielectric Materials, Measurements and Applications

EE Conf. Dept. Savoy Place, London WC2R OBL Ab: 27.10 .78 / P: 2.4.79

10-14 Sept.

Portoroz, Yugoslavis

Int. Conf, on Mössbauer Spectroscopy

D. Hanzel, J. Stefan Institute, University of Ljubljana, POB 199, YU-61001 Ljubljan A: May 79/P: June 79/200/US \$ 80. -

\section{0-14 Sept.}

Poitiers, France

IUTAM Symposium: Optical Methods in Mechanics of Solids

A. Lagarde, Laboratoire de Mécanique des Solides, Université de Poitiers, 40, avenue du Recteur Pineau, F-86022 Poitiers

10-14 Sept.

Tucson, AZ, USA

Symposium on Atomic Spectroscopy (SAS-79) John A. Stoner, Jr., Dept. of Physics, University of Arizona, Tucson, AZ 85721

10-15 Sept.

Lancaster, UK

2nd European Crystal Growth Conference (ECCG-2)

F.W. Ainger, Secretary ECCG-2, Allen Clark Research Centre, The Plessey Company Ltd., Caswell, Towcester, NN12 8EQ

13-19 Sept.

Batavia, IL, USA

13-19 Sept. Helen Peterson, Fermi National Accelerato inv. aboratory, POB 500, Batavia, IL 60510

17-21 Sept.

4ème Colloque Spécialisé Ampère:

Leipzig, GDR Dynamic Processes in Molecular Systems as studied by RF-Spectroscopy

A. Lösche, Physikalisches Institut der K. Marx Universität, Linnéstrasse 5, DDR-701 Leipzig C1 A: 1.6.79 / Ab: 15.4 .79 /PP / 200 / US \$ 50.

17-21 Sept.

Canterbury, UK

3rd Europhysics Topical Conf. on Lattice Defects in Ionic Crystals

The Secretary, Europhysics Conference,

Physics Laboratory, The University,

Canterbury CT2 7NR

A: 31.5.79/PP: 31.3.79 / $300 / £$ 50. -

17-21 Sept.

26th Int. Symposium on Macromolecules POB 900440, D-6000 Frankfurt 90

17-21 Sept.

Oxford, UK

9th European Conf. on Controlled Fusion and Plasma Physics

J.H.C. Maple, Culham Laboratory

Abingdon OX14 3DB

A: $1.7 .79 / A b: 1.3 .79 / P: 1.7 .79 / 450$

23-26 Sept.

Geneva, Switzerland

3rd World Telecommunication Forum - Technica Symposium

International Telecommunication Union.

$\mathrm{CH}-1211$ Geneva 20

Ab: 30.9 .78

25-26 Sept.

Nottingham, UK

Autumn Meeting of the British Radiofrequency Spectroscopy Group

P. Mansfield, Dept. of Physics, University of Nottingham

25-27 Sept. Phases

A. Keller, H.H. Wills Physics Laboratory, Royal Fort, Tyndall Avenue, Bristol BS8 1TL

A: Aug. 79 / PP: 1.5.79/150

October

San Diego, CA, USA

Octic

F.J. Nichols, Lectro Magnetics, Inc., 6056 West Jefferson Blvd., Los Angeles, CA 90016

7-12 Oct. 
23-25 Oct.

11 th Nat: Technical MA, USA SAMPE

30 Oct.-2 Nov.

Berkeley, CA, USA

8th Symposium on Engineering Problems of Fusion Research

T.H. Batzer, Lawrence Livermore Laboratory,

POB 808, Livermore, CA 94550

12-16 Nov.

Boston, MA, USA

Meeting of Plasma Physics Division (APS)

American Physical Society, 335 E. 45th Street New York, NY 10017

19-21 Nov.

West Lafayette, IN, USA

Meeting of Fluid Dynamics Division (APS)

American Physical Society, 335 E. 45th Street New York, NY 10017

Late Nov./Early Dec. $\quad$ Cambridge, MA, USA Annual Meeting of High Energy Astrophysics Division (American Astronomical Society)

Robert Novick, Department of Physics, Columbia University, 538 W. 12th Street, New York, NY 10017

3-5 Dec.

Washington, DC, USA

International Electron Devices Meeting

Technical Activities Board, IEEE, 345 E. 47th Street, New York, NY 10017

1000

10-12 Dec. Houston, TX, USA Meeting of Electron and Atomic Physics Division (APS)

merican Physical Society, 335 E. 45th Street New York, NY 10017

10-15 Dec.

Miami Beach, FL, USA

nfrared and Submillimetre Waves

K.J. Button, MIT National Magnet Lab.

170 Albany St., Cambridge, MA 02139

Ab: $2.8 .79 / 300$

\section{0}

13-15 Feb.

San Francisco, CA, USA

Int. Solid State Circuits Conference

Lewis Winner, 301 Almeria Avenue, Coral Gables, FL 33134

Spring

Jerusalem, Israel

5 th Int. Congress: International Radiation Protection Association

T. Schlesinger, IAEC, Soreq Nuclear Research Centre, Yavne 70600

\section{1-25 April}

Int. Magnetics Conference (INTERMAG)

D.I. Gordon, Naval Surface Weapons Center. White Oak Lab., Silver Springs, MD 20901

19-22 May

Madison, Wisconsin, USA

Int Conference on Plasma Science

Technical Activities Board, IEEE, 345 E. 47th Street, New York, NY 10017

27-31 May Washington, DC, USA

International Microwave Symposium

Boris Sheleg, Naval Research Laboratory, Code 5258. Washington, DC 20375

8-13 June Las Vegas, Nevada, USA Annual National Meeting: American Nuclear Society, David G. Pettengill, American Nuclear Society, 555 North Kensington Ave.

La Grange Park, IL 60525

6-10 July

Grenoble, France

Int. Conf.: Liquides et Amorphes Métalliques

F. Cyrot-Lackmann, Groupe des Transitions de Phases, CNRS, Avenue des Martyrs, $166 \mathrm{X}$ F-38042 Grenoble Cedex

\section{7-22 Aug.}

Santa Cruz, CA, USA

5th IUPAC Conf. on Physical Organic Chemistry

J.F. Bunnett, Division of Natural Sciences - II,

University of California, Santa Cruz, CA 95064 IUPAC

\section{8-22 Aug. \\ Micro 80}

Brighton, UK

The Administrator, Royal Microscopical Society 37/38 St. Clements, Oxford OX4 1AJ
24-30 Aug.

The Hague, NL

7 th European Congress on Electron Microscop aboratory for Electron Microscopy, University of Leyden, Rijnsburgerweg 10, Leyden

25-30 Aug.

Delft, NL

21 st Congress Ampère

7 th ISMR Symposium

J. Smidt, Laboratorium voor Technische Natuurkunde, Lorentzweg 1, Delft

Sept. or Oct.

8th Int. Vacuum Congress

4 th Int. Conf. on Solid Surfaces

D.A. Degras, Centre d'Etudes Nucléaires de Saclay, BT 62, B.P. No. 2, F-91190 Gif-sur-Yvette 1000 / IUVSTA

21-27 Sept.

Cannes, France

Surface Science Conf (ECOSS 3)

7-9 Oct. Baltimore, MD, USA

Electromagnetic Compatibility Symposium

A. Farrar, IIT Research Institute, ECAC / North Severn, Annapolis, MD 21402

9-15 Oct.

Düsseldorf, FRG

8th INTERKAMA

Arbeitsgemeinschaft INTERKAMA, Postfach 700969. D-6000 Frankfurt/Main

29-31 Oct.

Atlanta, Georgia, USA

Sympoia: Nuclear Science, Nuclear Power Systems Techical Activities Board IEEE, 345 E. 47th Street, New York, NY 10017

10-14 Nov.

San Diego, CA, USA

Meeting of Plasma Physics Division (APS)

The American Physical Society, 335 E. 45th Street New York, NY 10017

11-14 Nov.

Dallas, Texas, USA

Magnetism and Magnetic Materials

D.C. Bullock. Texas Instruments Inc. РОВ 5936 MS 145, Dallas, TX 75222

8-10 Dec.

Washington, DC, USA

Int. Electron Devices Meeting

Technical Activities Board, IEEE, 345 E. 47th Street, New York, NY 10017

\section{1}

\section{1-13 March}

Particle Accelerator Conference

Washington, DC, USA

Technical Activities Board, IEEE, 345 E. 47th Street, New York, NY 10017

April

Grenoble, France

International Magnetics Conference (INTERMAG)

D. Randet, CEN, B.P. No. 85, Centre de Tri, F-38041 Grenoble Cedex

15-17 June

Int. Microwave Symposium

Clavin, Hughes Aircraft Company, Bldg. 268, 8433 Fallbrook Ave., Canoga Park, CA 91304

2-6 Nov.

Washington, DC, USA

Meeting of Plasma Physics Division (APS)

The American Physical Society, 335 E. 45th Street, New York, NY 10017

\section{Schools \\ 1978}

Application forms for free places at the Erice schools may be obtained on request from the EPS Secretariat in Geneva.

10-21 July Stanford, CA, USA

On Particle Physics - Weak Interactions - Present and Future

Martha C. Zipf, Stanford Linear Accelerator Center, POB 4349 , Bin 62, Stanford, CA 94305 A: $30.4 .78 /$ US $\$ 75$. -
17-28 July

ISABELLE Summer Workshops

Upton, NY, USA

J. Alspector, Bldg. 510-A, Brookhaven National Laboratory, Upton, NY 11973

24 July-5 Aug.

Albany, NY, USA

Physics and Applications of Ion Beam Interaction with Solids

W.M. Gibson, Dept. of Physics, State University, Albany, NY 12222

24 July-2 Sept.

La Jolla, CA, USA

Summer Workshop: Deep Underwater Muon and Neutrino Detection

A. Roberts, DUMAND, Fermilab, POB 500

Batavia, IL 60510

31 July-11 Aug. 4

Erice, Italy

Int. School of Subnuclear Physics: The New Aspects of Subnuclear Physics

A. Zichichi, CERN, $\mathrm{CH}-1211$ Geneva 23

A: 20.7 .78 / Sfr. 1000 .

31 July-18 Aug.

La Jolla, CA, USA

Summer Workshop in Elementary Particles

La Jolla Institute, POB 1434, La Jolla, CA 92038

6-19 Aug.

Waterloo, Ontario, Canada

5th Course on Solar Energy Conversion

Solar Energy Conversion, Dept. of Physics, University of Waterloo, Waterloo, Ont., Canada N2L 3GI A: 30.6.78/PP / US \$250.-

6-20 Aug. Antigonish, Nova Scotia, Canada Group Theory for Spectroscopy

J.C. Domini, St. Francis Xaver University, Antigonish, NS, Canada B2G 1 CO

6-26 Aug. St. Andrews, UK

Metal Non-Metal Transition in Disordered Systems

7-18 Aug.

Cambridge, UK

Globular Clusters

M.J. Rees, Inst. of Astronomy, Madingley Road, Cambridge $\mathrm{CB} 3 \mathrm{OHA}$

11-20 Aug.

Erice, Italy

Interdisciplinary Seminar: The Meaning of Culture inv.

13-18 Aug.

Oberwolfach, FRG

* Mathematical Methods in Celestial Mechanics

14-19 Aug.

Pécz, Hungary

* Int. Summer School: Diffraction Studies on Noncrystalline Substances

15-30 Aug.

Arles, France

* Electronic and Magnetic Distributions in Molecules and Crystals

18 Aug.-15 Sept.

* Ecole d'Eté de Physique Spatiale

20 Aug.-2 Sept.

rasbourg, France Radiation applied to Biophysical, Biomedical and Biochemical Research

21 Aug.-2 Sept.

Banff, Canada

Nuclear Theory

B. Castel, Physics Dept., Queen's University, Kingston, Ont., Canada K7L 3N6

22-31 Aug.

Athens, Greece

* IR and Raman Spectroscopy of Biological Molecules

27 Aug.-30 Sept.

Uppsala, Sweden

* Int. Summer Institute in Quantum Chemistry and Solid State Physics

28 Aug.-2 Sept.

Cavtat, Yugoslavia

* 9th Summer School and Symposium on the Physics of lonized Gases — SPIG 78

28 Aug.-8 Sept.

nr. Auch, France

Interlinking of Computer Networks

K.G. Beauchamp, Computer Services Department, University of Lancaster, Bailrigg, Lancs, UK A: Feb. 78 / Ab: March 78/PP: March 78/70/inv.

28 Aug.-9 Sept.

Ghent, Belgium

Electrons in Disordered Metals and at Metallic Surfaces

28 Aug.-10 Sept. 4 Mikolajki, Poland * 11 th Summer School on Nuclear Physics

29 Aug.-6 Sept.

Ecole d'Eté d'Astronomie de Digne

Mlle L. Gouguenheim, Radioastronomi Observatoire de Meudon, F-92190 Meudon

* For full details please see March 1978 issue of Europhysics News 


\section{L'ÉCOLE POLYTECHNIQUE FÉDÉRALE DE LAUSANNE}

met au concours un poste de

\section{Professeur}

\section{de physique métallurgique}

\section{pour son Département de physique.}

\section{Délai d'inscription : 30 septembre 1978}

Les personnes intéressées voudront bien demander le dossier relatif à ce poste à la :

Direction Administrative de l'Ecole Polytechnique Fédérale de Lausanne, 33, avenue de Cour, 1007 Lausanne/Suisse.
3-15 Sept.

Aussois, France

P. Costa, OM - ONERA, 29 avenue de la Division Leclerc, F-92320 Châtillon

4-14 Sept.

Rhodes, Greece

5th Ampere Int. Summer School: Nuclear Resonance in Solids

F. Milia, Nuclear Research Centre 'Demokritos',

A: 31.5.78 / Ab: 31.5.78/100/English, French / US \$ 50 . -

4-16 Sept. Varenna, Italy

Diagnostics for Controlled Fusion Experiments E. Sindoni, Istituto di Fisica, 16 Via Celoria, I-20133 Milan

A: $30.6 .78 / 80 /$ US $\$ 150$.

4-16 Sept.

Karlsruhe, FRG

Advanced Summer Institute: New Phenomena in Lepton-Hadron Physics

D.C. Fries, Inst. für Theoretische Physik, Universität Karlsruhe, Kaiserstr. 12, D-7500 Karlsruhe PP / 100

10-22 Sept. Predeal, Romania Predeal International School in Physics - Heavy Ion Physics

I.A. Dorobantu, Central Institute of Physics, POB 5206, Bucharest

A: 15.7.78 / Ab: 15.7.78/PP / Lei 500.— incl. proc.

11-22 Sept.

Urbino, Italy

* Organometallics of the f-Elements - Chemical and Physical Properties of Lanthanide and Actinide Organometallic ' Compounds

12-22 Sept.

Maria Laach, FRG

2-22 Sept. für Hochenergiephysik: Eichtheorien und ihre experimentellen Grundlagen

17 Sept.-1 Oct.

Kupari, Yugoslavia

21 st Int. School of Elementary Particle Physics

Henri Braun, Division des Hautes Energies, C.B.H Centre de Recherches Nucléaires,

F-67037 Strasbourg-Cedex

A: $10.5 .78 / \mathrm{NP} / 80$ / Sfr. 700 .-

18-26 Sept.

Erice, Italy

Int. School of Fusion Reactor Technology: Driven Magnetic Fusion Reactors

B. Brunelli, Laboratori Nazionali dei Gas Ionizzati, CNEN, c.p. 65, 1-00044 Frascati

A: 15.7.78 / PP / 70 / US \$ 210. — all incl.

18-30 Sept. Primorsko,
*12th Int. School on High Energy Physics

Primorsko, Bulgaria à l'Etat Solide Aghia Paraskevi, Attiki
19-29 Sept.

Erice, Italy

Int. School of Meteorology of the Mediterranean A. Nania, Ufficio Ricerche Operative Meteorologia Aeronautica, Aeroporto, I-72100 Brindisi

9-20 Oct.

Trieste, Italy Theories of Condensed Matter

Manuel de Llano, Instituto de Fisica, Apdo. 20-364, Mexico 20, D.F., Mexico

13-21 Oct.

Erice, Italy

Workshop on Theoretical Physics - Hadronic Matter at Extreme Energy Density

N. Cabibbo, Université Pierre et Marie Curie, Tour 16 4 place Jussieu, F-75230 Paris Cedex 05

15-22 Oct.

Erice, Italy Int. School of Logic and Scientific Methodology . Agazzi, Istituto di Filosofia, Piazza S. Sabina 2 |-16124 Genoa

25 Oct.-3 Nov.

Erice, Italy

Int. School of Radiation Damage and Protection: The Use of Computers in Health Physics: Calculational Techniques in Shielding and Dosimetry

A. Rindi, INFN-LNF, c.p. 13, I-00044 Frascati A: 15.8 .78 / US $\$ 250$.

25 Oct:-23 Nov.

Trieste, Italy

Autumn Course on Systems Analysis: Theory, Methods and Applications

The Deputy Director, International Centre for Theoretical Physics, POB 586, I-34100 Trieste

13-16 Dec.

Erice, Italy Int. School of Applied Geophysics: Int. Symposium inv. of Hydrogeology

\section{9}

23 Jan.-30 March

Trieste, Italy Winter College on Atomic and Molecular Physics and Quantum Optics

The Deputy Director, International Centre for Theoretical Physics, POB 586, I-34100 Trieste A: 31.8 .78

\section{3-21 March}

Erice, Italy

Highly Specialized Seminar

G. Preparata, CERN, CH-1211 Geneva 23

25 March-5 Apri

Erice, Italy

Int. School of Physics of Exotic Atoms

G. Torelli, Istituto Nazionale di Fisica Nucleare, Via Vecchia Livornese, I-56010 San Piero a Grado Pisa 2nd Latin American Workshop on Selfconsistent

Int. School of Nuclear Physics: Heavy Ion Interactions at High Energies

D.H. Wilkinson, University of Sussex, Sussex House Falmer, Brighton BN1 9RH, UK

22-29 April

Erice, Italy

Int. School of History of Science

Y. Cappelletti, Istituto della Enciclopedia Italiana, Piazza Paganica 4, I-00186 Rome

6-18 May

Erice, Italy

Int. School of Cosmology and Gravitation: Spin, Torsion, Rotation and Supergravity

V. De Sabbata, Istituto di Fisica "A. Righi», Via Irnerio 46, I-40126 Bologna

1-17 June

Erice, Italy

Int. School of Atomic and Molecular Spectroscopy

B. Di Bartolo, Boston College, Dept. of Physics, Chestnut Hill, MA 02167, USA

11-25 June

Erice, Italy

Int. School of Low Temperature Physics: Phase Transitions in Systems of Reduced Dimensionality

T. Regge, Istituto di Fisica, University of Turin, Corso M. d'Azeglio 46, I-10125 Turin

1-14 July

Erice, Italy

Int. School of Astrophysics: X-Ray Astronomy

G.C. Setti, Istituto di Fisica «A. Righi», Via Irnerio 46 I-40126 Bologna

30 July-17 Aug.

St. Andrews, UK

20th Scottish Universities Summer School in Physics - Laser Plasma Interactions

J. Cumming, Department of Natural Philosophy, University of Glasgow, Glasgow, G12 800

1-10 Aug.

Int. School of Subnuclear Physics

Erice, Italy

A. Zichichi, CERN, CH-1211 Geneva 23

27 Aug.-8 Sept.

Varenna, Italy

Physics of Plasmas close to Thermonuclear Conditions

E. Sindoni, Istituto di Fisica, Via Celoria 16 I-20133 Milano

30 Aug.-9 Sept.

Madrid, Spain

Jù Aug.de Surface et la Liaison Chimique

Marcel Bourg, U.E.R. de Physique, Université de Provence, Place V. Hugo, F-13331 Marseille Cedex 3

Ab: 30.2.79 / NP / 100 / French, Italian, Spanish English

31 Aug.-8 Sept.

Durham, UK

International Summer School on the Characterization of Crystal Growth Defects by X-Ray Methods

B. Tanner, Department of Physics, University of Durham, Science Laboratories, South Road, Durham DH1 3LE

PP

1-15 Sept.

Erice, Italy

Int. School of Energetics: Technology in the Field of Energetics

F. Amman, Istituto di Fisica Applicata dell' Università, Via A. Bassi 6, I-27100 Pavia

16-26 Sept.

Erice, Italy

Int School of Radiation Damage and Protection A. Rindi, INFN-LNF, c.p. 13, I-00044 Frascati

23-31 Oct.

Erice, Italy

23-31 Oct.

G. Preparata, CERN, CH-1211 Geneva 23

24-28 Oct. Erice, Italy Int. School of Logic and Scientific Methodology E. Agazzi, Istituto di Filosofia, Piazza S. Sabina 2, I-16124 Genoa

Editor: E.N. Shaw

Meetings Compilation: W.S. Newman

Editorial Board

G.J. Béné, B. Hauck, G.R. Macleod,

J. Muller, D. Pohl, J.A. Schwarzmüller

All correspondence to:

Editor, EUROPHYSICS NEWS

European Physical Society.

P.O. Box 69

CH-1213 Petit-Lancy 2,

Phone: Geneva 931130

Switzerland

Published by the European Physical Society

Printed by: Ed. Cherix et Filanosa SA CH-1260 Nyon, Switzerland

* For full details please see March 1978 issue of Europhysics News 